Check for updates

Cite this: RSC Adv., 2018, 8, 23947

\title{
20(S)-Ginsenoside Rg2 attenuates myocardial ischemia/reperfusion injury by reducing oxidative stress and inflammation: role of SIRT1
}

\author{
Wenwen Fu,,$^{\mathrm{a}}$ Huali Xu, $\dagger^{\mathrm{a}}$ Xiaofeng $\mathrm{Yu},{ }^{\mathrm{a}}$ Chen Lyu, ${ }^{\mathrm{a}}$ Yuan Tian, ${ }^{\mathrm{a}}$ Minyu Guo, ${ }^{\mathrm{a}}$ \\ Jiao Sun ${ }^{\star b}$ and Dayun Sui $(\mathbb{D}$ *a
}

Previously we demonstrated that 20(S)-ginsenoside Rg2 protects cardiomyocytes from $\mathrm{H}_{2} \mathrm{O}_{2}$-induced injury by inhibiting reactive oxygen species (ROS) production, increasing intracellular levels of antioxidants and attenuating apoptosis. We explored the protective effect of 20(S)-ginsenoside Rg2 on myocardial ischemia/reperfusion (MI/R) injury and to clarify its potential mechanism of action. Rats were exposed to 20(S)-ginsenoside Rg2 in the presence/absence of the silent information regulator SIRT(1) inhibitor EX527 and then subjected to MI/R. 20(S)-Ginsenoside Rg2 conferred a cardioprotective effect by improving post-ischemic cardiac function, decreasing infarct size, reducing the apoptotic index, diminishing expression of creatine kinase-MB, aspartate aminotransferase and lactate dehydrogenase in serum, upregulating expression of SIRT1, B-cell lymphoma-2, procaspase-3 and procaspase-9, and downregulating expression of Bax and acetyl (Ac)-p53. Pretreatment with 20(S)-ginsenoside Rg2 also resulted in reduced myocardial superoxide generation, gp $91^{\text {phox }}$ expression, malondialdehyde content, cardiac pro-inflammatory markers and increased myocardial activities of superoxide dismutase, catalase and glutathione peroxidase. These results suggested that MI/R-induced oxidative stress and inflammation were attenuated significantly by $20(S)$-ginsenoside Rg2. However, these protective effects were blocked by EX527, indicating that SIRT1 signaling may be involved in the pharmacological action of 20(S)ginsenoside $\mathrm{Rg} 2$. Our results demonstrated that 20(S)-ginsenoside $\mathrm{Rg} 2$ attenuates $\mathrm{MI} / \mathrm{R}$ injury by reducing oxidative stress and inflammatory responses via SIRT1 signaling.

Received 16th March 2018 Accepted 11th June 2018 DOI: $10.1039 / c 8 \mathrm{ra02316f}$ rsc.li/rsc-advances

\section{Introduction}

Myocardial infarction is a major cause of mortality worldwide. Although early reperfusion therapy is necessary for myocardial salvage, reperfusion triggers "myocardial ischemia/reperfusion” (MI/R). MI/R is characterized by cardiomyocyte apoptosis, cardiac hypofunction, arrhythmia and other disorders, ${ }^{1,2}$ which exacerbate myocardial damage. ${ }^{3}$

Several factors contribute to the development of MI/R injury: cellular calcium loading; "no reflow" due to cell swelling; impaired vascular relaxation; and formation of "white cell plugs". The enhanced oxidative stress observed after reperfusion, however, is believed to be the most likely cause. ${ }^{4}$ Oxidative stress leads to reactive oxygen species (ROS) production, increases in malondialdehyde (MDA) content, and subsequent cytotoxic injury. ${ }^{4,5}$ These processes play key parts in the progression of

${ }^{a}$ Department of Pharmacology, School of Pharmaceutical Sciences, Jilin University, Changchun 130021, China. E-mail: suidy@jlu.edu.cn

${ }^{b}$ School of Nursing, Jilin University, Changchun, 130021, China. E-mail: sunjiao@jlu. edu.cn

$\dagger$ These authors contributed equally to this work and should be considered co-first authors. ischemic heart disease, and can contribute to cardiomyocyte apoptosis. ${ }^{5,6}$ Subsequently, ROS trigger leukocyte chemotaxis and cause inflammation, further aggravating severe cardiac damage., ${ }^{4,7}$ Increasing evidence suggests that ROS accumulation in mitochondria can lead to apoptotic cell death. ${ }^{8}$ ROS may also have direct effects on the structure and function of cells, which can lead to myocardial remodeling and might develop into heart failure., ${ }^{910}$ Therefore, protecting cardiomyocytes from ROS damage could be a rational method for ameliorating $\mathrm{MI} / \mathrm{R}$ injury.

Ginsenosides are the major active components of ginseng. The latter has a range of pharmacologic and therapeutic uses, including vasorelaxation as well as anti-neoplastic, anti-diabetes mellitus, anti-inflammatory and anti-oxidative effects. ${ }^{11-16} 20(S)$ Ginsenoside $\mathrm{Rg} 2$ is a major active protopanaxatriol-type compound present in the roots and stem leaves of ginseng (Fig. 1). Recent studies have shown that 20(S)-ginsenoside Rg2 can block calcium channels and displays activity against free radicals. ${ }^{17}$ Our recent study demonstrated that $20(S)$-ginsenoside $\mathrm{Rg} 2$ protects cardiomyocytes from $\mathrm{H}_{2} \mathrm{O}_{2}$-induced injury by increasing the intracellular levels of antioxidants, reducing ROS production, and subsequent apoptosis. ${ }^{18}$ However, few studies have explored the anti-oxidative and anti-inflammatory effects of 20(S)-ginsenoside $\mathrm{Rg} 2$ in MI/R injury. 


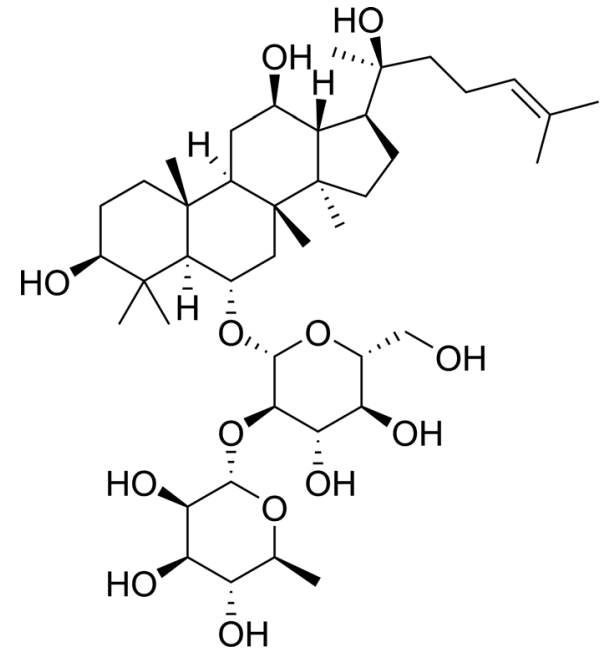

Fig. 1 Chemical structure of 20(S)-ginsenoside Rg2.

Silent information regulator (SIRT) 1 is a type of histone deacetylase, the activity of which is dependent on nicotinamide adenine dinucleotide $\left(\mathrm{NAD}^{+}\right)$. SIRT1 regulates the acetylation of p53, thereby inhibiting cardiomyocyte apoptosis. ${ }^{19}$ Studies have shown that activation of SIRT1 signaling protects against MI/R injury ${ }^{20,21}$ It has been demonstrated that SIRT1 expression is reduced after MI/R-induced injury, and that activation of SIRT1 signaling with curcumin, melatonin or berberine confers protection against $\mathrm{MI} / \mathrm{R}$-induced injury by suppressing oxidative stress. ${ }^{22-24}$ Our recent study showed that $20(S)$-ginsenoside $\mathrm{Rg} 2$ can protect $\mathrm{H} 9 \mathrm{c} 2$ cells against $\mathrm{H}_{2} \mathrm{O}_{2}$-induced injury through its anti-oxidative and anti-apoptotic actions. ${ }^{18}$ Conversely, ginsenosides Rb1, Rc, Rh3, 20(S)-ginsenoside Rg3 and extract of red ginseng can activate SIRT1..$^{25-30}$ In particular, 20(S)-ginsenoside Rg2 and some of its metabolites have high potential as SIRT1 activators, such as resveratrol. ${ }^{31}$ EX527 is a highly specific inhibitor of SIRT1 which suppresses NAD-dependent deacetylation in various cell types and conditions. ${ }^{32,33}$ Presumably, EX527 can be used to identify if SIRT1 can mediate the cardioprotective effect of $20(S)$-ginsenoside $\mathrm{Rg} 2$, and whether it relates to oxidative stress and inflammation through inhibition of the SIRT1 activation triggered by $20(S)$-ginsenoside Rg2.

We wished to ascertain if $20(S)$-ginsenoside Rg2 confers antioxidative and anti-inflammatory effects in $\mathrm{MI} / \mathrm{R}$, and to investigate the role of SIRT1 signaling in the cardioprotective effect of 20(S)-ginsenoside Rg2.

\section{Materials and methods}

\section{Chemicals and reagents}

20(S)-Ginsenoside Rg2 was provided by Professor Yifa Zhou (School of Life Sciences, Northeast Normal University, Changchun, China). High-performance liquid chromatography showed that the purity of $20(S)$-ginsenoside $\mathrm{Rg} 2$ was $>98 \%$. It was dissolved in $0.5 \%$ sodium carboxymethyl cellulose (CMC$\mathrm{Na}$ ) to give the final concentrations.

EX527 was purchased from Selleck Chemicals (Houston, TX, USA). Creatine kinase-MB (CK-MB), aspartate aminotransferase
(AST), lactate dehydrogenase (LDH), MDA, catalase (CAT), superoxide dismutase (SOD), glutathione peroxidase (GSH-PX), interleukin (IL)- $1 \beta$, IL-6 and tumor necrosis factor (TNF)- $\alpha$ assay kits were purchased from Nanjing Jiancheng Bioengineering Institute (Nanjing, China). The terminal deoxynucleotidyl transferase-mediated dUTP nick end labeling (TUNEL) assay using an in situ cell death detection kit was obtained from Roche Molecular Biochemicals (Mannheim, Germany). A quantitative polymerase chain reaction (qPCR) kit was obtained from Beijing TransGen Biotech (Beijing, China). A bicinchoninic acid (BCA) protein assay kit was purchased from Beyotime Institute of Biotechnology (Jiangsu, China). Antibodies against SIRT1, acetyl (Ac)-p53, gp91 ${ }^{\text {phox }}$, procaspase-3, procaspase-9, Bcell leukemia (Bcl)-2 and Bax were purchased from Cell Signaling Technology (Beverly, MA, USA). Antibody against $\beta$ actin was obtained from ZSGB-BIO (Beijing, China). Goat antirabbit and goat anti-mouse secondary antibodies were purchased from Beijing Dingduo Changsheng Biotechnology (Beijing, China).

\section{Animals}

Animals were treated according to the Guide for the Care and Use of Laboratory Animals (US National Institutes of Health (NIH), Bethesda, MD, USA) and the Committee for the Care and Use of Laboratory Animals of Jilin University (Changchun, China). The study protocol was approved by the Ethics Committee of Jilin University.

Female Sprague-Dawley rats $(230-250 \mathrm{~g})$ were provided by the Experimental Animal Center of Jilin University. All animals were allowed free access to food and water, and maintained at 22-24 ${ }^{\circ} \mathrm{C}$ under a $12 \mathrm{~h}$ light-dark cycle.

\section{Experimental protocol}

Step 1. We wished to investigate if pretreatment with $20(S)$ ginsenoside Rg2 alleviated MI/R-induced injury. Rats were assigned randomly to four experimental groups, and given agents via the intragastric route. In the sham group, rats were administered $0.5 \%$ CMC-Na (10 mL kg ${ }^{-1}$ body weight). In the $\mathrm{MI} / \mathrm{R}$ group, rats were administered $0.5 \% \mathrm{CMC}-\mathrm{Na}\left(10 \mathrm{~mL} \mathrm{~kg}^{-1}\right)$. In the $\mathrm{MI} / \mathrm{R}+\mathrm{Rg} 210 \mathrm{mg} \mathrm{kg}{ }^{-1}$ group, rats were administered $20(S)$-ginsenoside Rg2 $\left(10 \mathrm{mg} \mathrm{kg}^{-1}\right)$. In the $\mathrm{MI} / \mathrm{R}+\mathrm{Rg} 220 \mathrm{mg}$ $\mathrm{kg}^{-1}$ group, rats were administered $20(S)$-ginsenoside Rg2 $\left(20 \mathrm{mg} \mathrm{kg}^{-1}\right)$. Rats were given agents once a day for 3 continuous days. One hour after administration on day 3 , rats were anesthetized for surgery.

Step 2. We wished to investigate the cardioprotective role of SIRT1 after pretreatment with 20(S)-ginsenoside Rg2 in MI/Rinduced injury. Rats were assigned randomly to four experimental groups. In the $\mathrm{MI} / \mathrm{R}$ group, rats were administered $0.5 \%$ CMC-Na (10 $\mathrm{mL} \mathrm{kg}^{-1}$, i.g.). In the $\mathrm{MI} / \mathrm{R}+\mathrm{Rg} 2$ group, rats were administered $20(S)$-ginsenoside $\mathrm{Rg} 2\left(20 \mathrm{mg} \mathrm{kg}^{-1}\right.$, i.g.). In the $\mathrm{MI} / \mathrm{R}+\mathrm{Rg} 2$ + EX527 (selective SIRT1 inhibitor) group, rats received 20(S)-ginsenoside Rg2 following the regimen mentioned above and EX527 (5 mg kg${ }^{-1}$, i.p.) every 2 days. In the MI/R + EX527 group, rats were administered EX527 (5 mg 
$\mathrm{kg}^{-1}$, i.p.) every 2 days. One hour after administration on day 3 , rats were anesthetized surgery.

\section{Experimental model of $\mathrm{MI} / \mathrm{R}$ injury}

Sprague-Dawley rats were anesthetized with $3 \%$ pentobarbital sodium. Myocardial ischemia was induced by exteriorizing the heart through a left-thoracic incision, placing a 6-0 silk suture and making a slipknot around the left anterior descending coronary artery. After $30 \mathrm{~min}$ of ischemia, the slipknot was released, and the myocardium reperfused for $6 \mathrm{~h}$ (for analyses of myocardial apoptosis and measuring the size of the myocardial infarct) and $72 \mathrm{~h}$ (for determination of cardiac function). Rats in the sham group underwent the same surgical procedures except that the suture passing under the left coronary artery was left untied. Before and during the surgical procedure, animals received different treatments.

\section{Echocardiography}

Two-dimensional (2D) and M-mode echocardiographic measurements were conducted in a standard setting using a $10 \mathrm{~S}$ scan head (Vivid I; GE Healthcare, Piscataway, NJ, USA) 72 h after MI/R. After a short-axis 2D image of the left ventricle had been obtained at the level of the papillary muscles, 2D-guided M-mode images were acquired at a sweep speed of $100 \mathrm{~mm}$ $\mathrm{s}^{-1}$ and stored digitally. The left ventricular internal dimension at diastole (LVIDd) and left ventricular internal dimension at systole (LVIDs) were measured, allowing calculation of left ventricular fractional shortening (LVFS) and left ventricular ejection fraction (LVEF; calculated using Simpson's rule). All parameters were measured over three consecutive cardiac cycles. Measurements were made by an experienced echocardiographer blinded to the treatment protocol.

\section{Determination of the size of the myocardial infarct}

At the end of $6 \mathrm{~h}$ of reperfusion, hearts were excised rapidly. Determination of infarct size was undertaken according to a method described previously with minor modification. ${ }^{34}$ Ventricle tissues were cut into five transverse slices, and the latter incubated in $0.5 \%$ nitrotetrazolium blue chloride for $20 \mathrm{~min}$. Myocardial infarction was distinguished by the different color (pale for ischemic myocardium and dark-red for non-ischemic myocardium). The ischemic myocardium was cut and weighed. The infarct size as a percentage of the ventricular mass was calculated as the weight of the ischemic zone/total weight of ventricle $\times 100 \%$. The size of the myocardial infarct was expressed as a percentage of the ventricle area.

\section{Determination of myocardial apoptosis}

To measure the DNA nicks that reflect the extent of myocardial apoptosis, the TUNEL assay was carried out. ${ }^{35}$ Heart slices were fixed in $10 \%$ formalin solution, embedded in paraffin, and sectioned on slides at $5 \mu \mathrm{m}$ thickness. TUNEL staining with paraffin sections was done according to manufacturer instructions. The apoptosis index was expressed as the number of apoptotic cardiomyocytes/total number of cardiomyocytes $\times$ $100 \%$.

\section{Determination of serum activities of CK-MB, AST and LDH}

Blood samples were drawn $6 \mathrm{~h}$ after reperfusion. Collected blood samples were clotted for $2 \mathrm{~h}$ at room temperature, and centrifuged at $900 \times g$ for $15 \mathrm{~min}$. The supernatant serums were separated and stored at $-80{ }^{\circ} \mathrm{C}$. The activities of CK-MB, AST and $\mathrm{LDH}$ were measured using diagnostic kits according to manufacturer instructions.

\section{Quantification of superoxide production}

Superoxide production in tissue was measured by lucigeninenhanced chemiluminescence, as described previously. ${ }^{36}$ One piece of cardiac tissue was weighed precisely. Phosphatebuffered saline containing phosphatase and protease inhibitors $(1: 9 \mathrm{w} / \mathrm{v})$ was added. After homogenization, the mixture was centrifuged at $145 \times g$ for $10 \mathrm{~min}$ at room temperature.

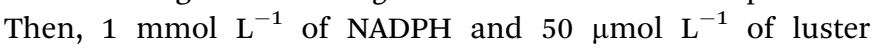
concentrate were added into the collected supernatant. A chemiluminescence analyzer was employed to measure the activity of ligninase. Superoxide production was expressed as relative light units per second per milligram heart weight $\left(\mathrm{RLU} \mathrm{mg}^{-1} \mathrm{~s}^{-1}\right)$.

\section{Determination of tissue MDA, SOD, CAT and GSH-PX}

The tissues of ventricles were excised and homogenized with ice-cold buffer in a homogenizer. The homogenate was centrifuged at $1780 \times g$ for $15 \mathrm{~min}$ at room temperature. The supernatant was taken to measure the activities of SOD, CAT and GSH-PX and MDA the content using diagnostic kits according to manufacturer instructions.

Table 1 Primer sequences

\begin{tabular}{llr}
\hline Gene & Primer sequence $\left(5^{\prime}-3^{\prime}\right)$ & Accession number \\
\hline IL-1 $\beta$ & Forward: GCAATGGTCGGGACATAGTT & NM_031512.2 \\
& Reverse: AGACCTGACTTGGCAGAGG & NM_012589.2 \\
IL-6 & Forward: AGTGCATCATCGCTGTTCATACA & \\
& Reverse: ATATGTTCTCAGGGAGATCTTGGAA & NM_012675.3 \\
TNF- $\alpha$ & Forward: GTCGTAGCAAACCACCAAGC & Neverse: TGTGGGTGAGGAGCACGTAG 031144.3 \\
$\beta-$ Actin & Forward: GATCAAGATCATTGCTCCTCCTG & Reverse: AGGGTGTAAAACGCAGCTCA
\end{tabular}




\section{Detection of tissue levels of IL-1 $\beta$, IL-6 and TNF- $\alpha$}

Rats were sacrificed after $6 \mathrm{~h}$ of reperfusion. Heart tissue was dissected rapidly for measurement of the mRNA levels and activities of IL-1 $\beta$, IL- 6 and TNF- $\alpha$. Total RNA was isolated from heart tissue using TRIzol ${ }^{\mathrm{TM}}$ reagent (Invitrogen, Carlsbad, CA, USA) according to manufacturer instructions. RNA was quantified by optical-density measurements at $260 \mathrm{~nm}$ and $280 \mathrm{~nm}$. Synthesis of complementary DNA and qPCR was carried out using TransScript ${ }^{\circledR}$ Green Two-Step qRT-PCR SuperMix (TransGen Biotech, Beijing, China). qPCR was done with a reaction mixture (total volume $=20 \mu \mathrm{L}$ ) that consisted of $2 \times$ Trans Start Top Green qPCR SuperMix, passive reference dye, double-distilled water, cDNA templates, as well as forward and reverse primers. The amount of mRNA from IL- $1 \beta$, IL- 6 and TNF- $\alpha$ was normalized to $\beta$-actin expression. All primers were ordered or custom-made from Beijing Dingguo Changsheng
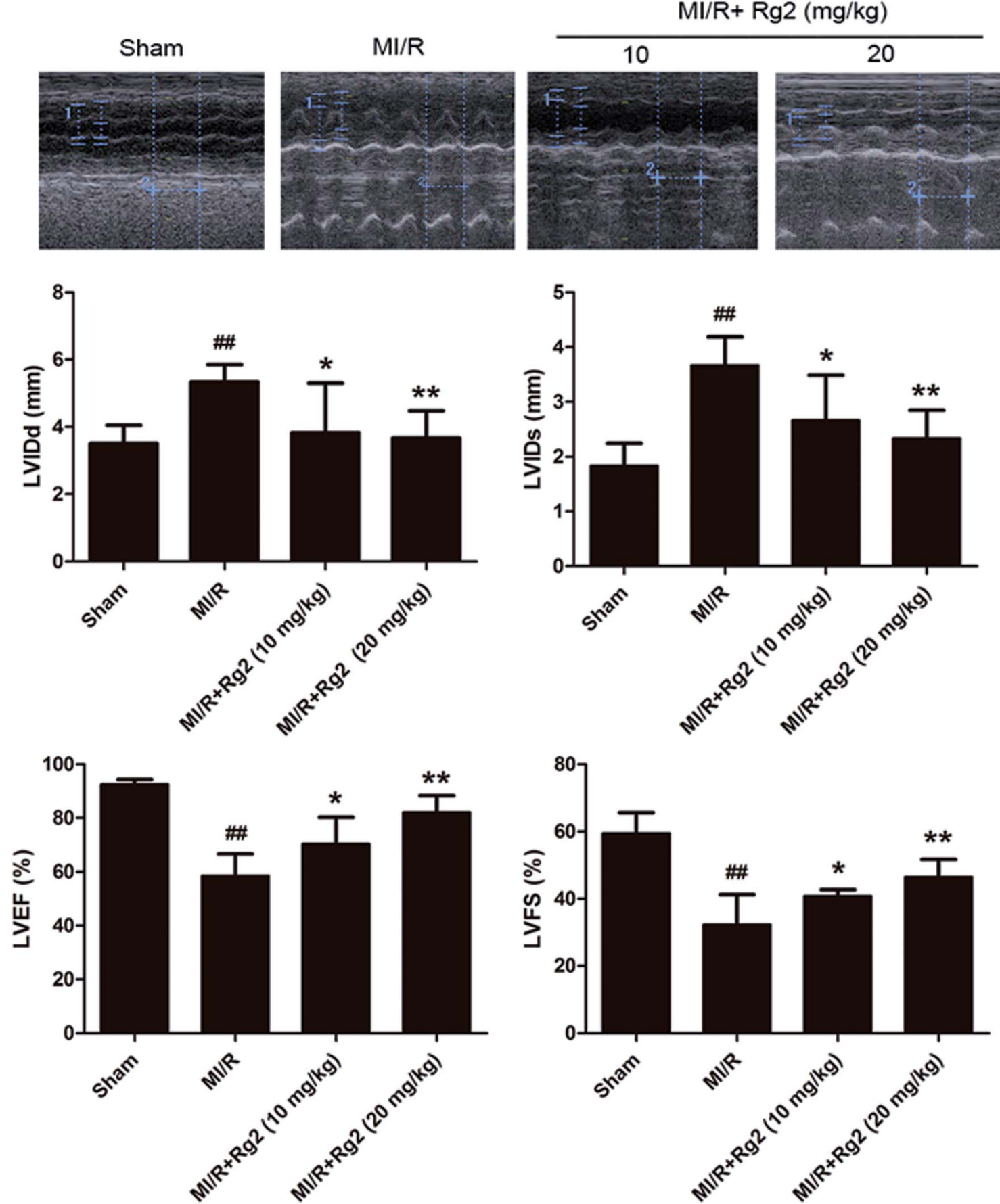

Fig. 2 Effects of 20(S)-ginsenoside Rg2 on cardiac function in MI/R-induced injury. Echocardiographic measurement was carried out after $72 \mathrm{~h}$ of reperfusion. Representative M-mode images are shown. Evaluation of left ventricular internal dimension at diastole (LVIDd), left ventricular internal dimension at systole (LVIDs), left ventricular ejection fraction (LVEF) and left ventricular fractional shortening (LVFS) are shown. Data are the mean \pm S.D. ${ }^{\# \#} P<0.01$ vs. sham group; ${ }^{*} P<0.05$, ${ }^{* *} P<0.01$ vs. MI/R group. 
Biotechnology (Table 1). Relative fold-changes in expression of the target gene in control and other groups were determined using the $2^{-\Delta \Delta C_{\mathrm{T}}}$ method. Activities of IL-1 $\beta$, IL- 6 and TNF- $\alpha$ in myocardial tissue homogenates and cardiomyocyte supernatants were detected in strict accordance with manufacturer instructions. ${ }^{37,38}$ A BCA kit was used to quantify protein.

\section{Western blotting}

Western blotting was used for detecting proteins from SIRT1, Ac-p53, gp91 ${ }^{\text {phox }}$, procaspase-3, procaspase-9, Bcl-2 and Bax. Rats were sacrificed after $6 \mathrm{~h}$ of reperfusion, and heart tissue was removed for protein extraction. The protein concentration was determined using the BCA protein assay kit. Then, the extracted protein was loaded onto $12 \%$ polyacrylamide-sodium dodecyl sulfate gel. After electrophoresis, the gel was blotted onto a polyvinylidene difluoride (PVDF) membrane and blocked with 5\% (w/v) non-fat milk for $1 \mathrm{~h}$. The transferred PVDF membrane was incubated with appropriate primary antibodies at $4{ }^{\circ} \mathrm{C}$ overnight. Binding of primary antibody was detected with a secondary antibody conjugated to horseradish peroxidase, and visualized using ECL chemiluminescence. $\beta$-Actin was used as the internal loading control. Results are expressed by grayscale value analyzed by Image J v1.5.0.26 (NIH).

\section{Statistical analyses}

Experiments were carried out at least thrice in duplicate/ triplicate. Results are the mean value \pm S.D. One-way ANOVA/ Bonfferroni and individual $t$-tests were conducted using Prism v5.04 (GraphPad, San Diego, CA, USA). $P<0.05$ was considered significant.
A
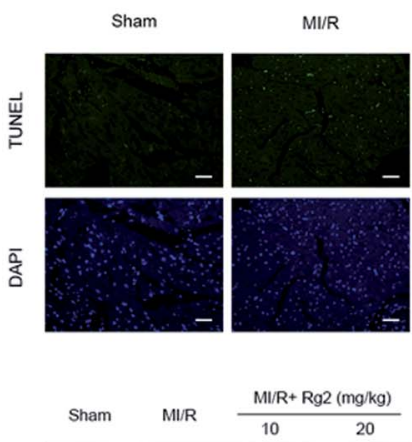

B
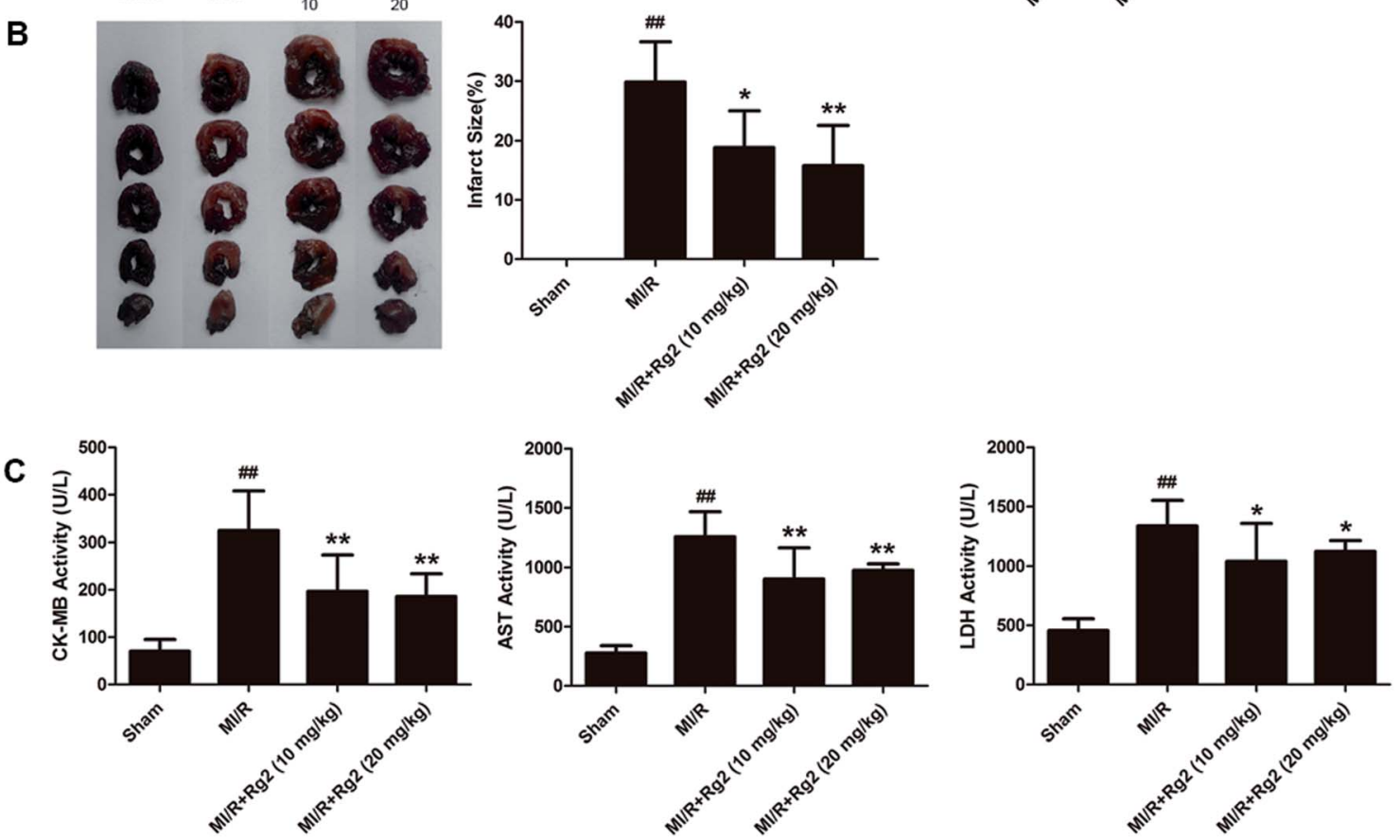

Fig. 3 Effects of 20(S)-ginsenoside Rg2 on the apoptotic index, infarct size, and serum activities of CK-MB, AST and LDH in MI/R-induced injury. Apoptotic index, infarct size, and serum levels of creatine-MB (CK-MB), aspartate aminotransferase (AST) and lactate dehydrogenase (LDH) were measured after $6 \mathrm{~h}$ of reperfusion. (A) Representative images of in situ detection of apoptotic cardiomyocytes by TUNEL staining. Green fluorescence shows TUNEL-positive nuclei; blue fluorescence shows nuclei of total cardiomyocytes (scale bar: $100 \mu \mathrm{m}$ ). (B) Representative photographs of heart sections. Pale color indicates an ischemic region and dark-red represents a non-ischemic region. The infarct size was calculated as a percentage of the ventricular mass: weight of ischemic zone/total weight of ventricle $\times 100 \%$. The size of the myocardial infarct is expressed as a percentage of the ventricle area. (C) Serum activities of CK-MB, AST and LDH after $6 \mathrm{~h}$ of reperfusion are shown. Data are the mean \pm S.D. ${ }^{\# \# P} 0.01$ vs. sham group; ${ }^{*} P<0.05, * * P<0.01$ vs. MI/R group. 
A

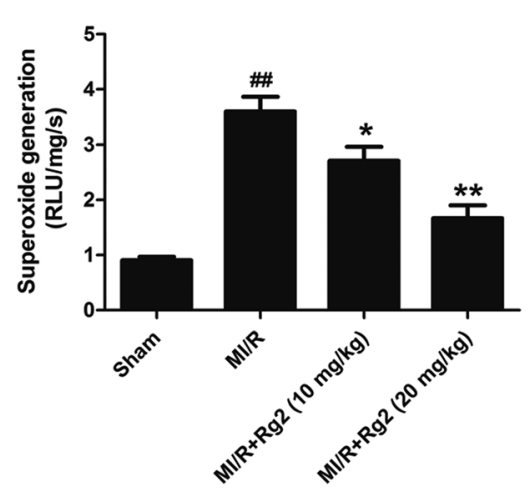

B

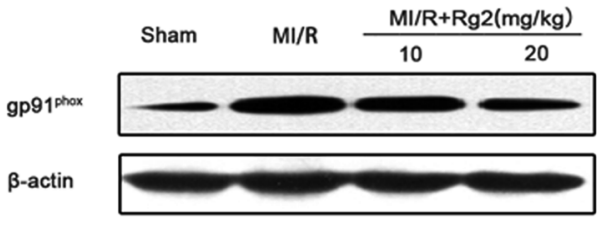

C
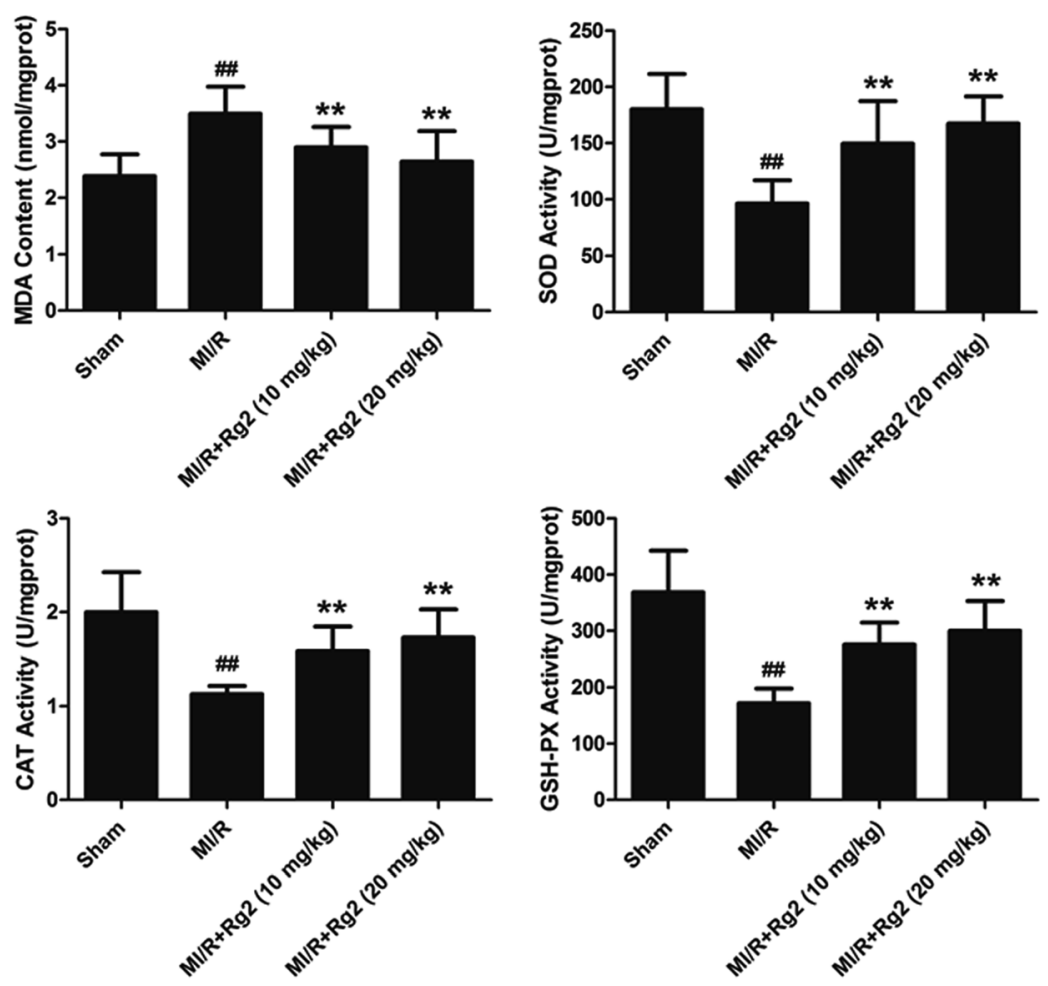

Fig. 4 Effects of 20(S)-ginsenoside Rg2 on oxidative stress in MI/R-induced injury. The level of cardiac oxidative stress was measured after $6 \mathrm{~h}$ of reperfusion. (A) Cardiac superoxide generation and (B) gp91 ${ }^{\text {phox }}$ expression were evaluated. (C) MDA content and the activities of SOD, CAT and GSH-PX in the myocardium were measured. Data are the mean \pm S.D. ${ }^{\# \#} P<0.01$ vs. sham group; $* P<0.05, * * P<0.01$ vs. MI/R group. 


\section{Results}

Effects of $20(S)$-ginsenoside $\mathrm{Rg} 2$ on cardiac function in $\mathrm{MI} / \mathrm{R}$ induced injury

After $72 \mathrm{~h}$ of reperfusion, echocardiography showed that cardiac function in the $\mathrm{MI} / \mathrm{R}$ group had deteriorated remarkably, as evidenced by increased LVIDd, LVIDs and decreased LVFS and LVEF compared with the sham group $(P<$ 0.01) (Fig. 2). Pretreatment with 20(S)-ginsenoside Rg2 (10 and $20 \mathrm{mg} \mathrm{kg}^{-1}$ ) ameliorated myocardial dysfunction significantly, which was verified by increased LVEF, LVFS and decreased LVIDd and LVIDs in MI/R animals $(P<0.05$ or $P<$ 0.01) (Fig. 2). Our results suggested that pretreatment with $20(S)$-ginsenoside $\mathrm{Rg} 2$ improved cardiac function in $\mathrm{MI} / \mathrm{R}$ induced injury.

Effects of 20(S)-ginsenoside Rg2 on the apoptotic index, infarct size, and serum activities of CK-MB, AST and LDH in MI/R-induced injury

After $6 \mathrm{~h}$ of reperfusion, pretreatment with 20(S)-ginsenoside $\mathrm{Rg} 2$ decreased cardiac apoptosis and necrosis by reducing the apoptotic index and infarct size compared with the MI/R group $(P<0.05$ or $P<0.01)$ (Fig. 3A and B). We evaluated further the activities of CK-MB, AST and LDH in serum, which reflected cellular injury or tissue necrosis and membrane permeability. Compared with the sham group, the activities of CK-MB, AST and $\mathrm{LDH}$ were increased in the MI/R group. However, pretreatment with $20(S)$-ginsenoside $\mathrm{Rg} 2\left(10\right.$ and $20 \mathrm{mg} \mathrm{kg}{ }^{-1}$ ) decreased the activities of CK-MB, AST and LDH $(P<0.05$ or $P<$
0.01) (Fig. 3C). These results provided direct evidence that $20(S)$ ginsenoside $\mathrm{Rg} 2$ could attenuate post-MI/R apoptosis and necrosis.

\section{Effects of $20(S)$-ginsenoside Rg2 on oxidative stress in $\mathbf{M I} / \mathbf{R}$ - induced injury}

Acute reperfusion enhances ROS generation. Therefore, we examined cardiac superoxide generation, expression of gp $91^{\text {phox }}$ protein, and oxidative parameters in heart homogenates. The $\mathrm{MI} / \mathrm{R}$ group showed a significant increase in superoxide content compared with the sham group (Fig. 4A), and pretreatment with $20(S)$-ginsenoside $\mathrm{Rg} 2$ (10 and $20 \mathrm{mg} \mathrm{kg}^{-1}$ ) decreased superoxide accumulation significantly $(P<0.05$ or $P<0.01)$. Then, we measured expression of gp91 ${ }^{\text {phox }}$, a major component of $\mathrm{NADPH}$ oxidase that is the most important superoxideproducing enzyme in the ischemic reperfused heart. $^{24}$ Compared with the MI/R group, pretreatment with $20(S)$-ginsenoside $\mathrm{Rg} 2$ (10 and $20 \mathrm{mg} \mathrm{kg}^{-1}$ ) decreased gp91 ${ }^{\text {phox }}$ expression markedly $(P<0.01)$ (Fig. 4B). The content of MDA (an index of lipid peroxidation) was increased significantly (Fig. 4C), whereas the activities of the antioxidant enzymes SOD, CAT and GSH-PX were decreased significantly in the $M I / R$ group compared with the sham group $(P<0.01)$. Pretreatment with 20(S)-ginsenoside $\mathrm{Rg} 2$ (10 and $20 \mathrm{mg} \mathrm{kg}^{-1}$ ) could decrease MDA content, and increase the activities of SOD, CAT, and GSH-PX ( $P$ $<0.01)$. These results showed that pretreatment with $20(S)$ ginsenoside Rg2 decreased superoxide production and reduced oxidative stress in MI/R-induced injury.
A

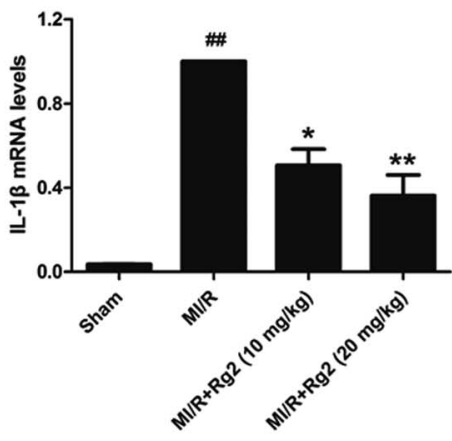

B

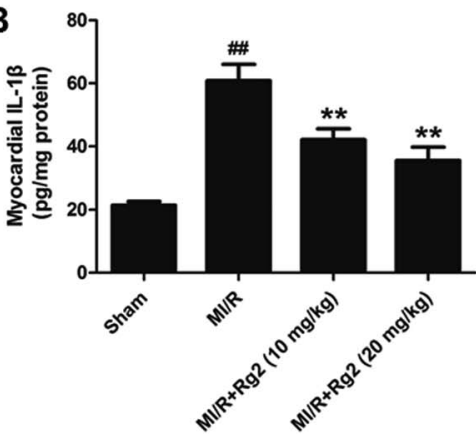

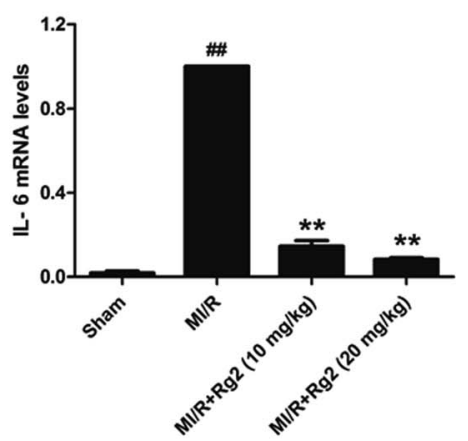
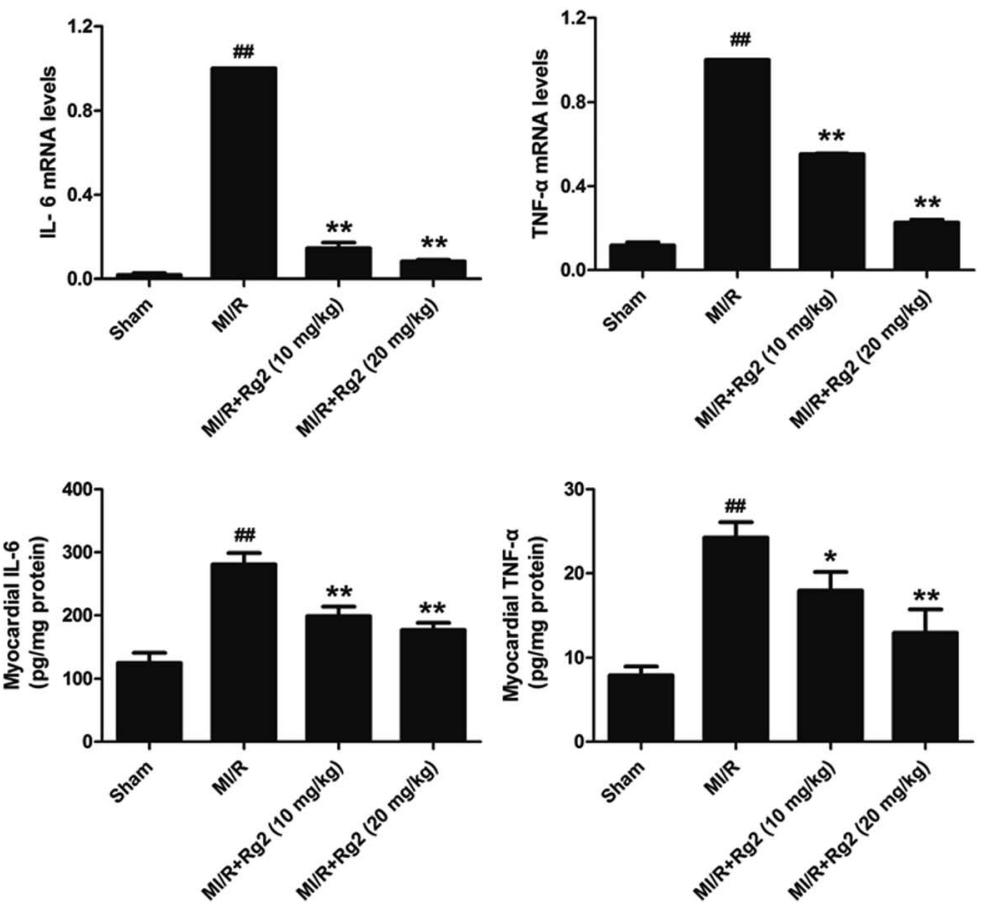

Fig. 5 Effects of 20(S)-ginsenoside Rg2 on the inflammatory response in MI/R-induced injury. (A) mRNA levels and (B) activities of IL-1 $1 \beta$, IL-6 and TNF- $\alpha$ in the myocardium were measured after $6 \mathrm{~h}$ of reperfusion. Data are the mean \pm S.D. $\# \# P<0.01 \mathrm{vs}$. sham group; $* P<0.05, * * P<0.01 \mathrm{vs.}$ $\mathrm{MI} / \mathrm{R}$ group. 

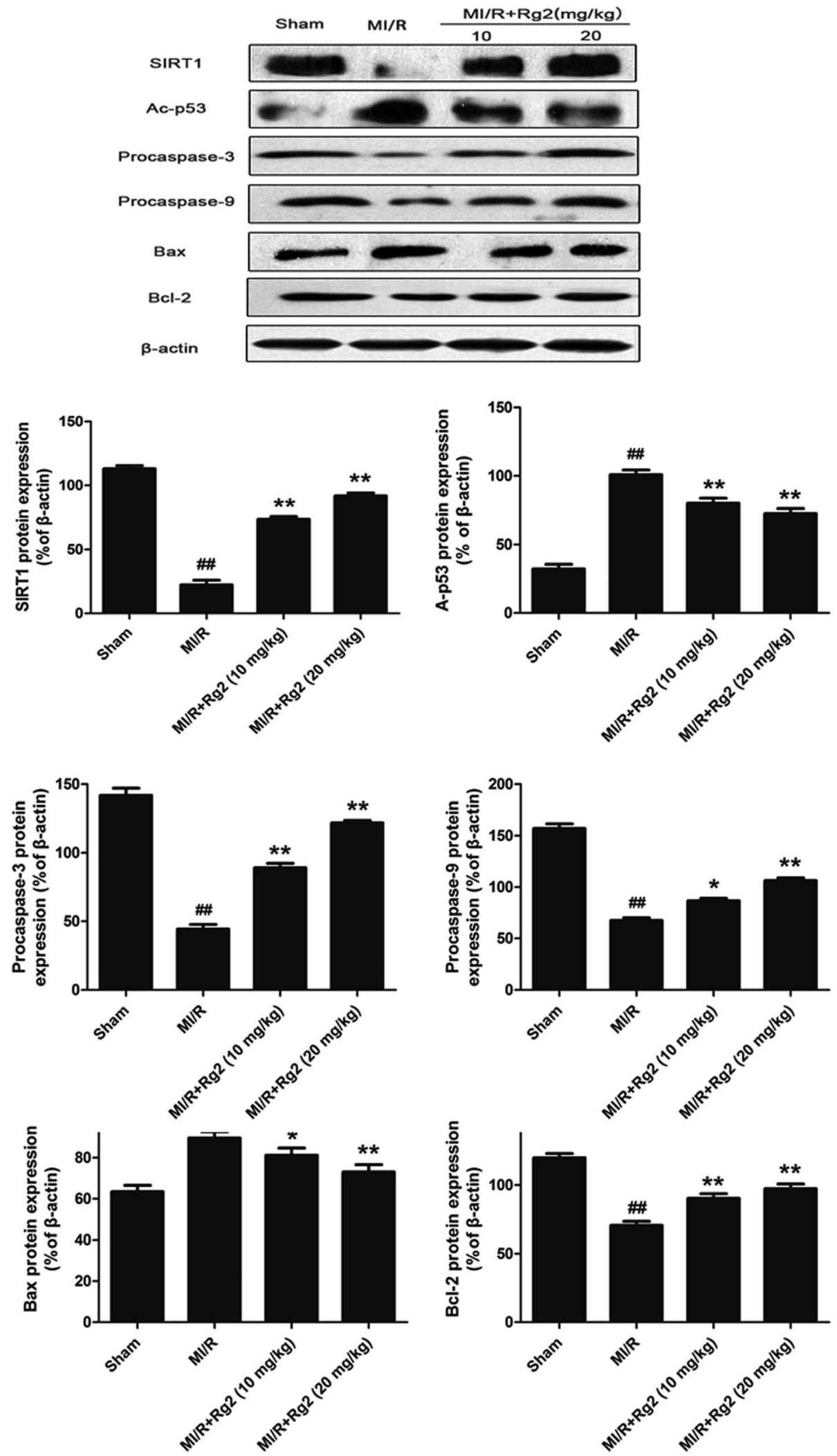

Fig. 6 Effects of 20(S)-ginsenoside Rg2 on expression of SIRT1, Ac-p53, procaspase-3, procaspase-9, Bax and Bcl-2 in MI/R-induced injury. SIRT1-related signaling and apoptosis-related protein levels were measured after $6 \mathrm{~h}$ of reperfusion. Representative blots of expression of SIRT1, Ac-p53, procaspase-3, procaspase-9, Bax and Bcl-2 are shown. Expression of the indicated proteins are shown as the mean \pm S.D. $\# \# P<0.01$ vs. sham group; $* P<0.05, * * P<0.01$ vs. MI/R group. 
Effects of $20(S)$-ginsenoside $\mathrm{Rg} 2$ on the inflammatory response in MI/R-induced injury

Pro-inflammatory cytokines (IL-1 $\beta$, IL-6 and TNF- $\alpha$ ) play an important part in the inflammatory response to MI/R injury. After $6 \mathrm{~h}$ of reperfusion, all the levels of pro-inflammatory factors were increased significantly in the $M I / R$ group compared with the sham group $(P<0.01)$ (Fig. 5). Compared with the $\mathrm{MI} / \mathrm{R}$ group, pretreatment with $20(S)$-ginsenoside Rg2 inhibited the increase in the mRNA levels and activities of IL-1 $\beta$, IL- 6 and TNF- $\alpha$ markedly $(P<0.05$ or $P<0.01)$. These results showed that pretreatment with $20(S)$-ginsenoside $\mathrm{Rg} 2$ reduced the inflammatory response in $\mathrm{MI} / \mathrm{R}$ induced injury.
Effects of 20(S)-ginsenoside Rg2 on expression of SIRT1, Acp53, procaspase-3, procaspase-9, Bcl-2 and Bax in MI/Rinduced injury

To examine the potential signaling pathways involved in the protective effects of $20(S)$-ginsenoside Rg2, we measured expression of SIRT1 and Ac-p53. SIRT1 expression was decreased significantly and Ac-p53 expression was increased significantly in the MI/R group compared with the sham group $(P<0.01)$ (Fig. 6). However, pretreatment with $20(S)$-ginsenoside Rg2 increased SIRT1 expression and decreased Ac-p53 expression compared with the MI/R group $(P<0.01)$. Next, we evaluated expression of apoptosis-related proteins. MI/R decreased the expression of procaspase-3, procaspase- 9 and $\mathrm{Bcl}-2$ but
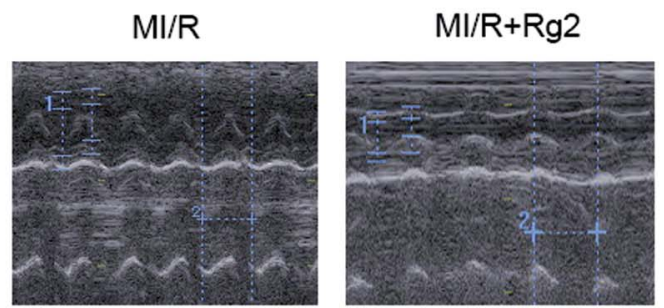

$M l / R+R g 2+E X 527$

$M I / R+E \times 527$
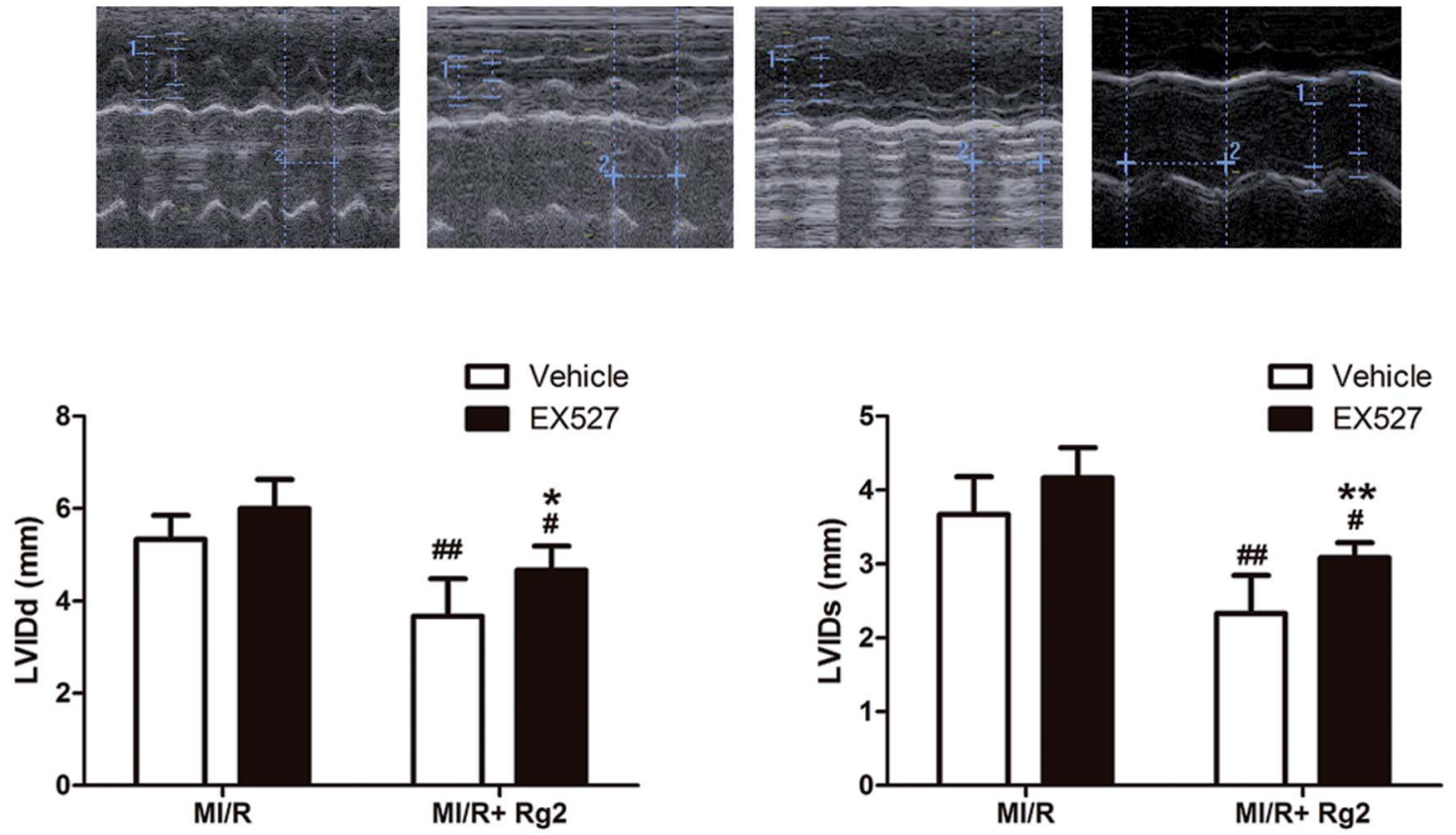

Vehicle

EX527
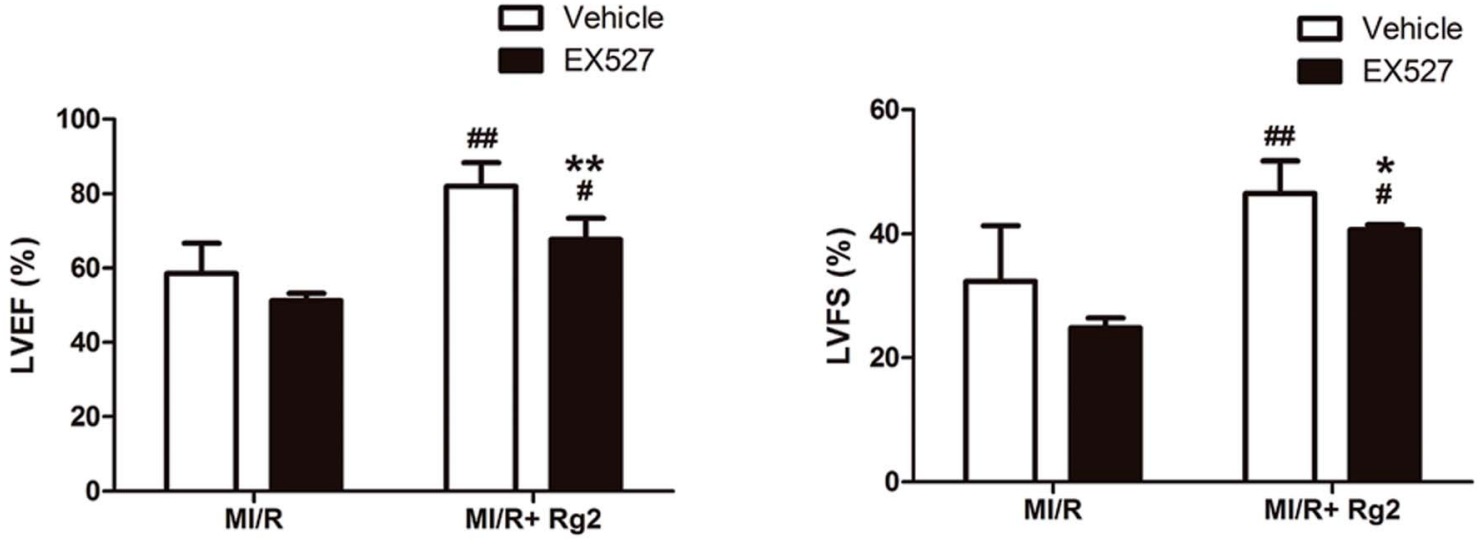

Fig. 7 Effects of 20(S)-ginsenoside Rg2 and EX527 on cardiac function in MI/R-induced injury. Echocardiographic measurement was carried out after $72 \mathrm{~h}$ of reperfusion. Representative M-mode images are shown. Evaluation of LVIDd, LVIDs, LVEF and LVFS is shown. Images of the MI/R group and MI/R + Rg2 group are shared with Fig. 2. Data are the mean \pm S.D. ${ }^{\#} P<0.05,{ }^{\# \#} P<0.01$ vs. MI/R group and $* P<0.05, * * P<0.01$ vs. MI/ $\mathrm{R}+\mathrm{Rg} 2$ group. 
increased the expression of Bax compared with the sham group $(P<0.01)$. In contrast, pretreatment with $20(S)$-ginsenoside Rg2 (10 and $20 \mathrm{mg} \mathrm{kg}^{-1}$ ) decreased the expression of Bax and increased the expression of procaspase-3, procaspase-9 and Bcl2 compared with the MI/R group $(P<0.05$ or $P<0.01)$. These results suggested that pretreatment with $20(S)$-ginsenoside $\mathrm{Rg} 2$ can activate SIRT1 expression, thus decreasing Ac-p53 expression, and inhibit an apoptotic signaling pathway.

\section{Effects of 20(S)-ginsenoside Rg2 and EX527 on cardiac function in MI/R-induced injury}

The results shown above suggested that the cardioprotective effect of 20(S)-ginsenoside $\mathrm{Rg} 2$ was associated with SIRT1 signaling. To investigate further the mechanisms underlying 20(S)-ginsenoside Rg2-induced cardioprotective effects, we introduced EX527, a selective SIRT1 inhibitor, ${ }^{32}$ to our study. Pretreatment with $20(S)$-ginsenoside $\mathrm{Rg} 2$ improved cardiac function significantly, as demonstrated by increased LVEF,
LVFS and decreased LVIDd and LVIDs compared with the MI/R group $(P<0.01)$ (Fig. 7). However, EX527 treatment abolished the protective effect of $20(S)$-ginsenoside $\mathrm{Rg} 2$ by decreasing LVIDd, LVIDs, LVEF and LVFS compared with the MI/R + Rg2 group $(P<0.05$ or $P<0.01)$, which suggested that the cardioprotective effect of $20(S)$-ginsenoside $\mathrm{Rg} 2$ may involve a SIRT1-related signaling pathway.

Effects of 20(S)-ginsenoside Rg2 and EX527 on the apoptotic index, infarct size, and serum activities of CK-MB, AST and LDH in MI/R-induced injury

We determined the apoptotic index, infarct size, and activities of CK-MB, AST, and LDH in serum. Pretreatment with $20(S)$ ginsenoside $\mathrm{Rg} 2$ could attenuate post-MI/R apoptosis and necrosis, which was demonstrated by reduction in the apoptotic index, infarct size, and activities of CK-MB, AST and LDH compared with the MI/R group $(P<0.01)$ (Fig. 8). However, the presence of EX527 abolished the protective effect of $20(S)$ -
A
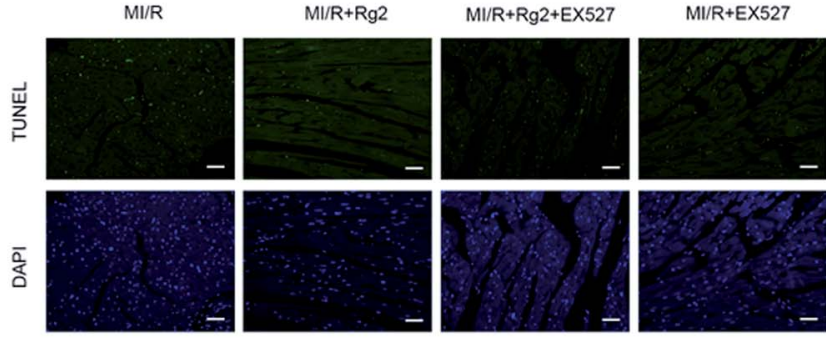

B MU/R MU/R+Rg2 MU/R+Rg2+EX527 ML/R+EX527

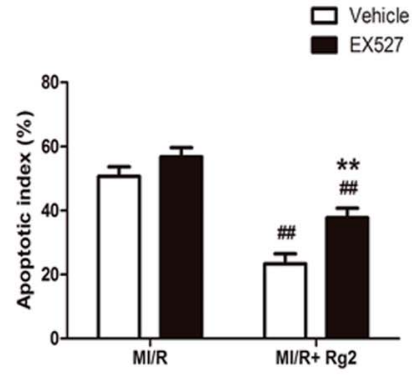

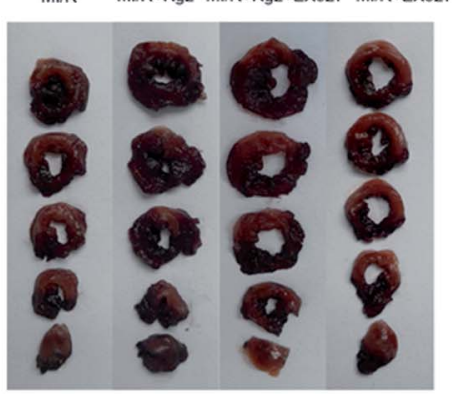
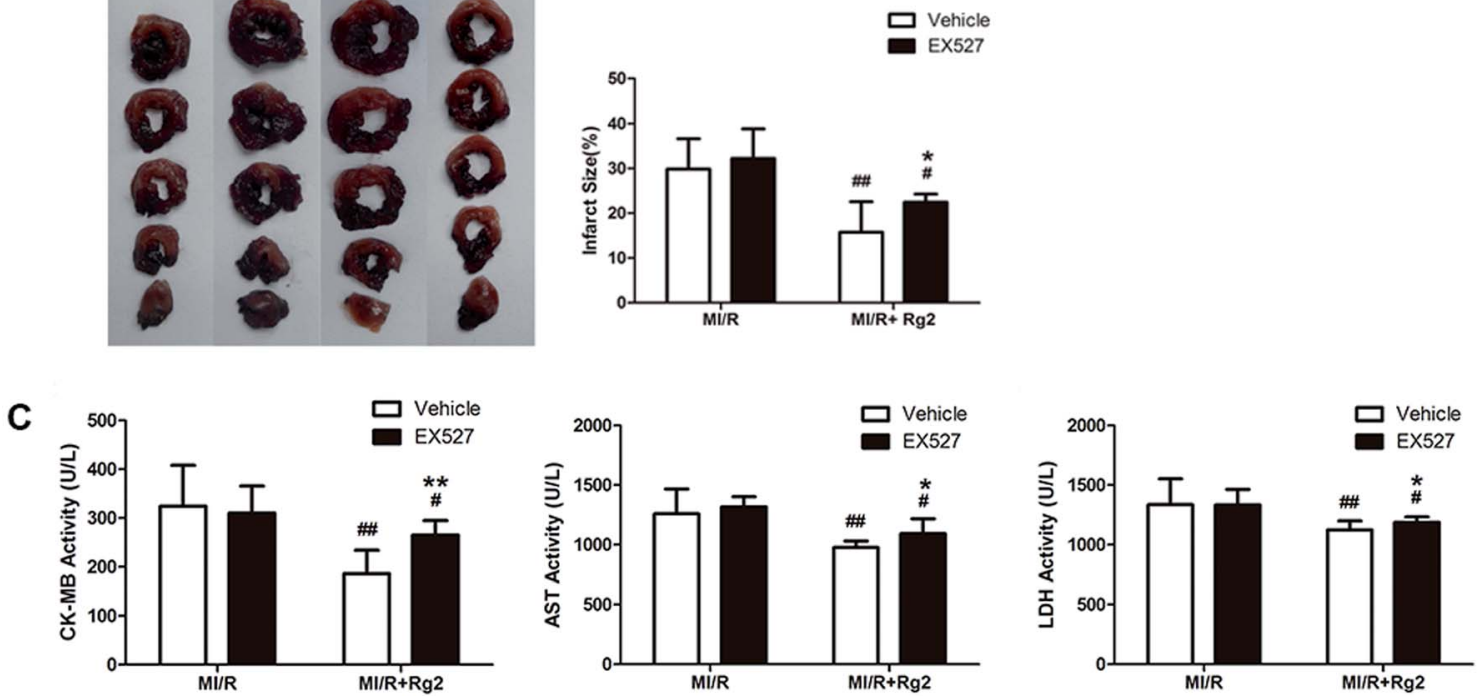

Fig. 8 Effects of 20(S)-ginsenoside Rg2 and EX527 on the apoptotic index, infarct size, and serum activities of CK-MB, AST and LDH in MI/Rinduced injury. Apoptotic index, infarct size, and serum activities of CK-MB, AST and LDH were measured after $6 \mathrm{~h}$ of reperfusion. (A) Representative images of in situ detection of apoptotic cardiomyocytes by TUNEL staining. Green fluorescence shows TUNEL-positive nuclei; blue fluorescence shows nuclei of total cardiomyocytes (scale bar: $100 \mu \mathrm{m}$ ). (B) The infarct size was calculated as a percentage of the ventricular mass: weight of ischemic zone/total weight of ventricle $\times 100 \%$. The size of the myocardial infarct is expressed as a percentage of the ventricle area. (C) The serum activities of CK-MB, AST and LDH after $6 \mathrm{~h}$ reperfusion are shown. Images from the MI/R group and MI/R + Rg2 group are shared with Fig. 3. Data are the mean \pm S.D. ${ }^{\#} P<0.05$ vs. Ml/R group and ${ }^{*} P<0.05,{ }^{* *} P<0.01$ vs. Ml/R + Rg2 group. 
ginsenoside $\mathrm{Rg} 2$ by increasing the apoptotic index, infarct size, the activities of CK-MB, AST and LDH in serum compared with the $\mathrm{MI} / \mathrm{R}+\mathrm{Rg} 2$ group $(P<0.05$ or $P<0.01)$. Our results suggested that the anti-post-MI/R effect of $20(S)$-ginsenoside $\mathrm{Rg} 2$ on apoptosis and necrosis was associated with activation of SIRT1 signaling.

A

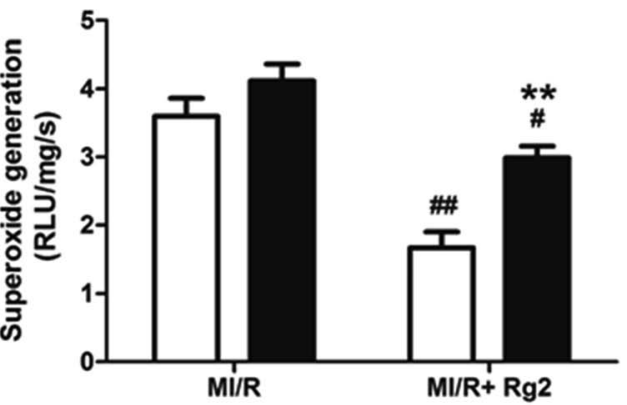

B $M I / R \quad M I / R+R g 2 \quad M I / R+R g 2+E X 527$ MIIR+EX527

gp919hox

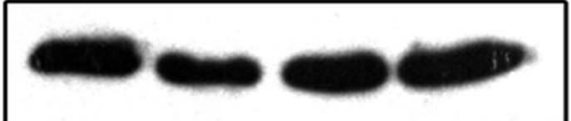

$\beta$-actin
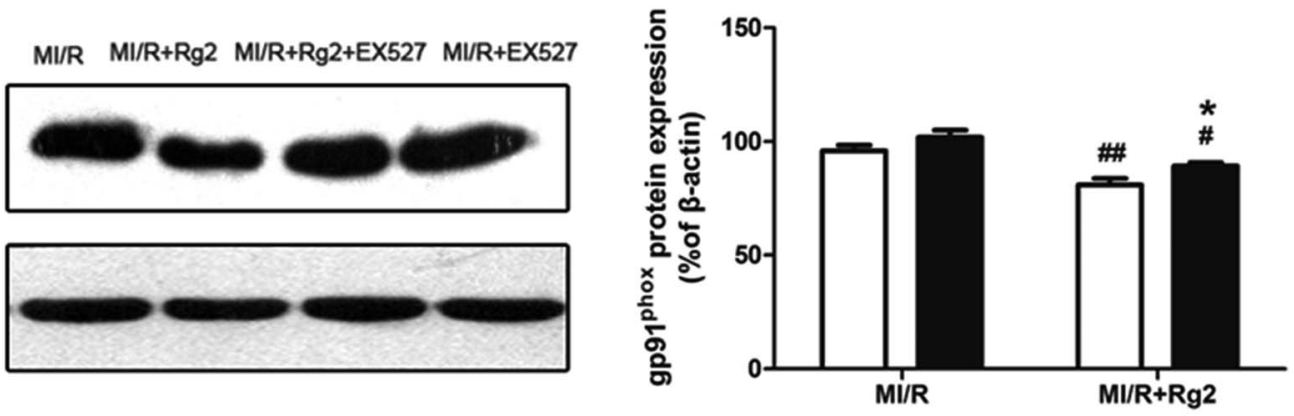

C
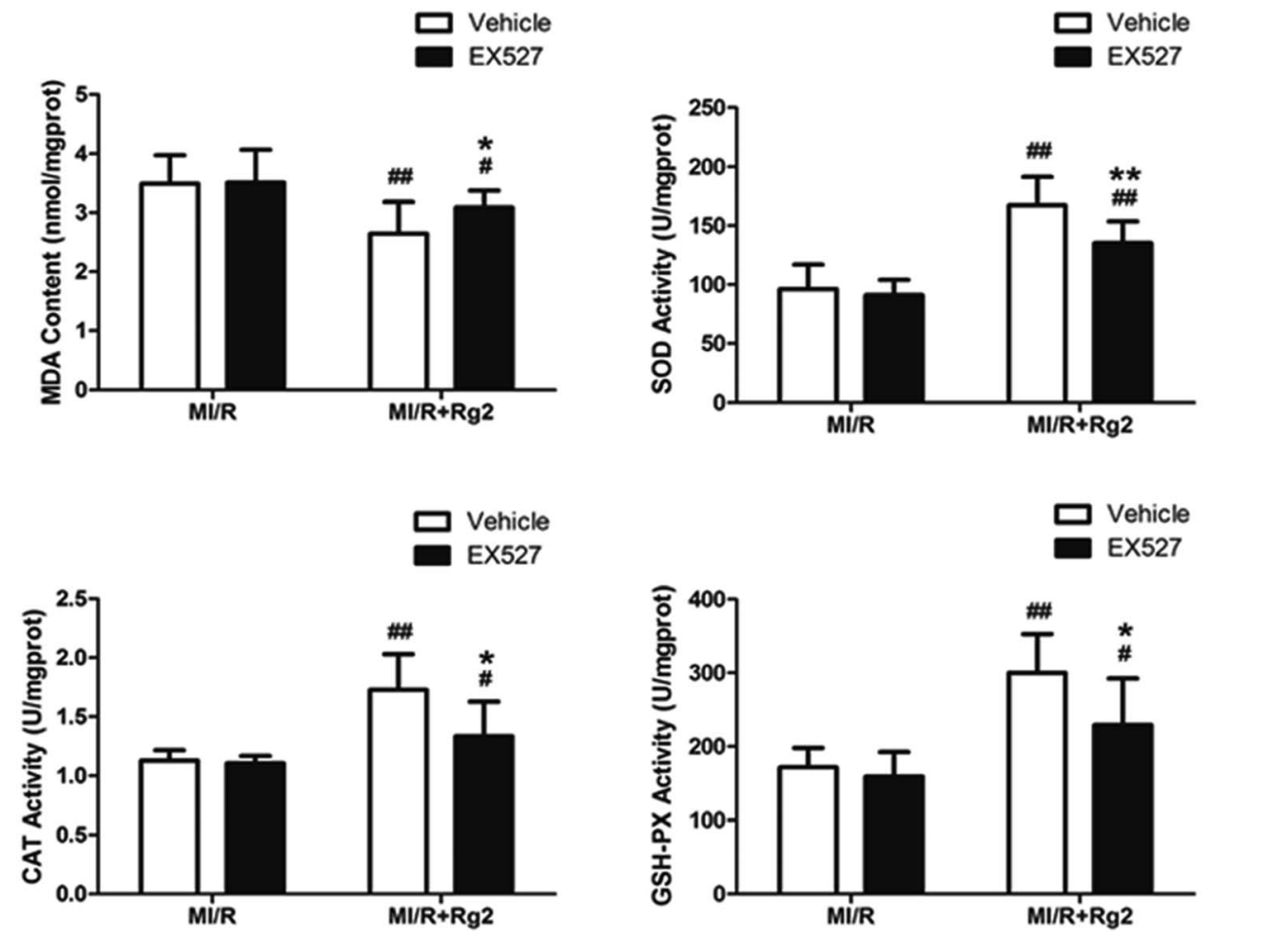

Fig. 9 Effects of 20(S)-ginsenoside Rg2 and EX527 on oxidative stress in MI/R-induced injury. Cardiac oxidative stress level was measured after $6 \mathrm{~h}$ of reperfusion. (A) Cardiac superoxide generation and (B) gp91 ${ }^{\text {phox }}$ expression were evaluated. (C) MDA content and activities of SOD, CAT and GSH-PX in the myocardium were measured. Data are the mean $\pm \mathrm{S}$. D. ${ }^{\#} P<0.05 \mathrm{vs}$. MI/R group and $* P<0.05, * * P<0.01 \mathrm{vs}$. MI/R $+\mathrm{Rg} 2$ group. 
Effects of 20(S)-ginsenoside Rg2 and EX527 on oxidative stress in $\mathrm{MI} / \mathrm{R}$-induced injury

As shown above, we demonstrated that pretreatment with $20(S)$-ginsenoside $\mathrm{Rg} 2$ decreased superoxide production and reduced oxidative stress in $\mathrm{MI} / \mathrm{R}$ hearts. We studied further the effect of EX527 on 20(S)-ginsenoside Rg2 treatment. 20(S)Ginsenoside Rg2-induced reductions in superoxide generation, gp91 ${ }^{\text {phox }}$ expression and MDA content were abolished upon EX527 treatment $(P<0.05$ or $P<0.01)$ (Fig. 9). The presence of EX527 also abolished the protective effect of $20(S)$ ginsenoside $\mathrm{Rg} 2$ by reducing the activities of SOD, CAT and GSH-PX in the myocardium compared with the MI/R + Rg2 group $(P<0.05$ or $P<0.01)$. Together with previous data obtained by our research team, ${ }^{\mathbf{1 8}}$ these results suggested that $20(S)$-ginsenoside $\mathrm{Rg} 2$ reduced post-MI/R oxidative stress, thereby improving cardiac function via a SIRT1-related signaling pathway.

\section{Effects of 20(S)-ginsenoside Rg2 and EX527 on the inflammatory response in $\mathrm{MI} / \mathrm{R}$-induced injury}

It has been demonstrated that $\mathrm{MI} / \mathrm{R}$ induces high expression of IL-1 $\beta$, IL- 6 and TNF- $\alpha$ in the myocardium, which aggravates myocardial damage. Pretreatment with 20(S)-ginsenoside Rg2 inhibited the increase in the mRNA levels and activities of proinflammatory cytokines markedly compared with the $\mathrm{MI} / \mathrm{R}$ group $(P<0.05$ or $P<0.01)$ (Fig. 10). However, EX527 treatment abolished the protective effect of $20(S)$-ginsenoside Rg2 by increasing the mRNA levels and activities of IL-1 $\beta$, IL-6 and TNF$\alpha$ compared with the $\mathrm{MI} / \mathrm{R}+\mathrm{Rg} 2$ group $(P<0.05)$. These results suggested that $20(S)$-ginsenoside $\mathrm{Rg} 2$ attenuated inflammation via a SIRT1 signaling pathway.
Effects of 20(S)-ginsenoside Rg2 and EX527 on expression of SIRT1, Ac-p53, procaspase-3, procaspase-9, Bcl-2 and Bax in MI/R-induced injury

To establish a more distinct relationship between $20(S)$-ginsenoside Rg2 and its anti-apoptotic property in MI/R injury, we investigated if EX527 administration could abolish the antiapoptotic effect of $20(S)$-ginsenoside $\mathrm{Rg} 2$. Pretreatment with 20(S)-ginsenoside Rg2 decreased the expression of Bax and increased the expression of procaspase-3, procaspase-9 and Bcl2 compared with the MI/R group $(P<0.01)$ (Fig. 11). However, EX527 treatment abolished the protective effect of $20(S)$-ginsenoside Rg2 by increasing Ac-p53 expression while decreasing SIRT1 expression compared with the MI/R + Rg2 group $(P<0.05$ or $P<0.01$ ). Consistent with previous observations, EX527 treatment enhanced apoptotic signaling by decreasing expression of procaspase-3, procaspase- 9 and Bcl- 2 while increasing Bax expression compared with the $\mathrm{MI} / \mathrm{R}+\mathrm{Rg} 2$ group $(P<0.05$ or $P<0.01)$. These results suggested that $20(S)$-ginsenoside $\mathrm{Rg} 2$ reduced apoptosis (at least in part) by upregulating SIRT1 expression.

\section{Discussion}

Here, we demonstrated that: (i) 20(S)-ginsenoside Rg2 protected against MI/R-induced injury by reducing oxidative stress and inflammation; (ii) the cardioprotective effect of $20(\mathrm{~S})$-ginsenoside Rg2 may (at least in part) be mediated by SIRT1 signaling. This is the first report demonstrating that $20(S)$-ginsenoside Rg2 protects against MI/R-induced injury by anti-oxidative and anti-inflammatory actions, and that these effects are associated with SIRT1 signaling.
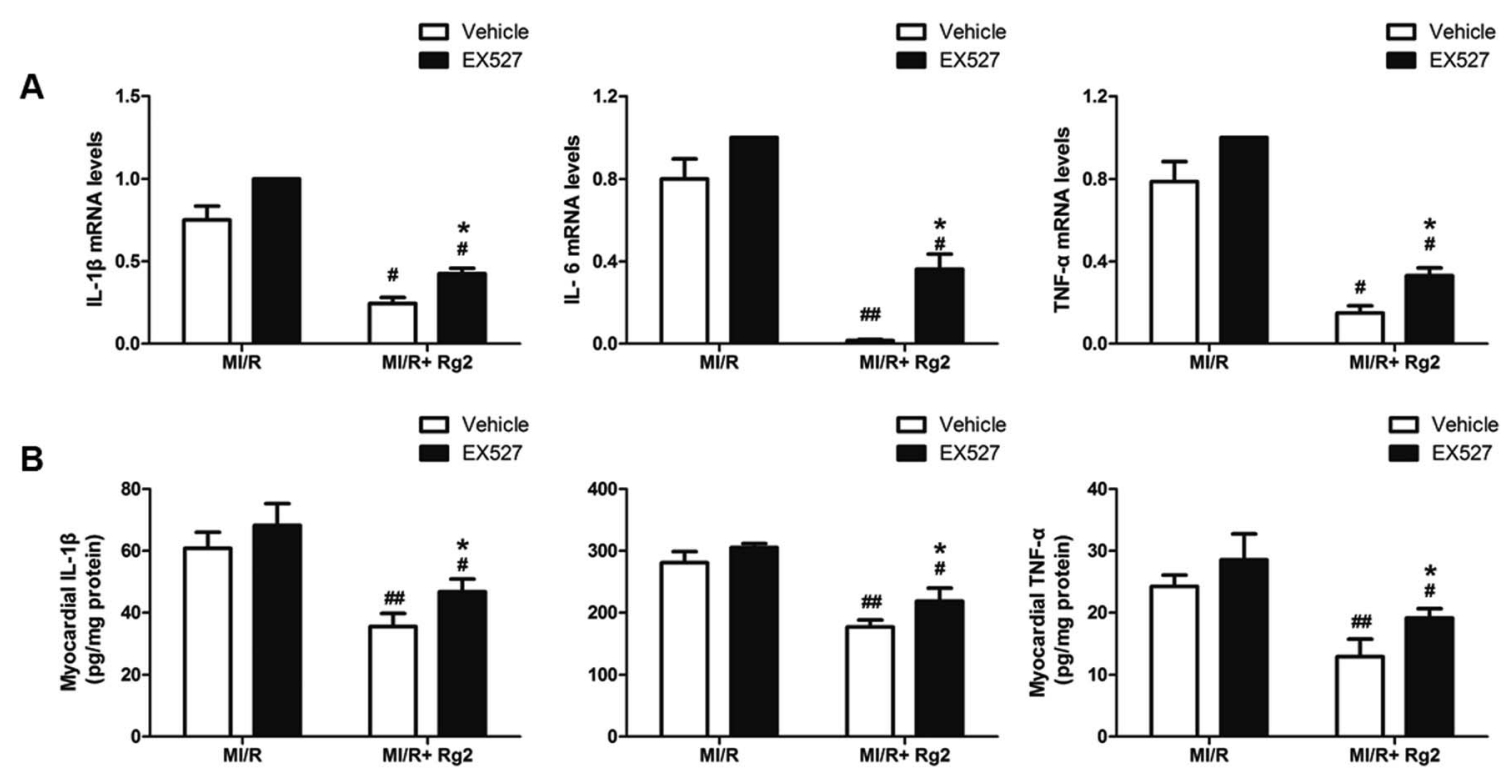

Fig. 10 Effects of 20(S)-ginsenoside Rg2 and EX527 on the inflammatory response in MI/R-induced injury. (A) mRNA levels and (B) activities of IL$1 \beta, I L-6$ and TNF- $\alpha$ in the myocardium were measured after $6 \mathrm{~h}$ of reperfusion. Data are the mean \pm S.D. ${ }^{\#} P<0.05,{ }^{\#} P<0.01$ vs. MI/R group and $* P<0.05$ vs. MI/R + Rg2 group. 

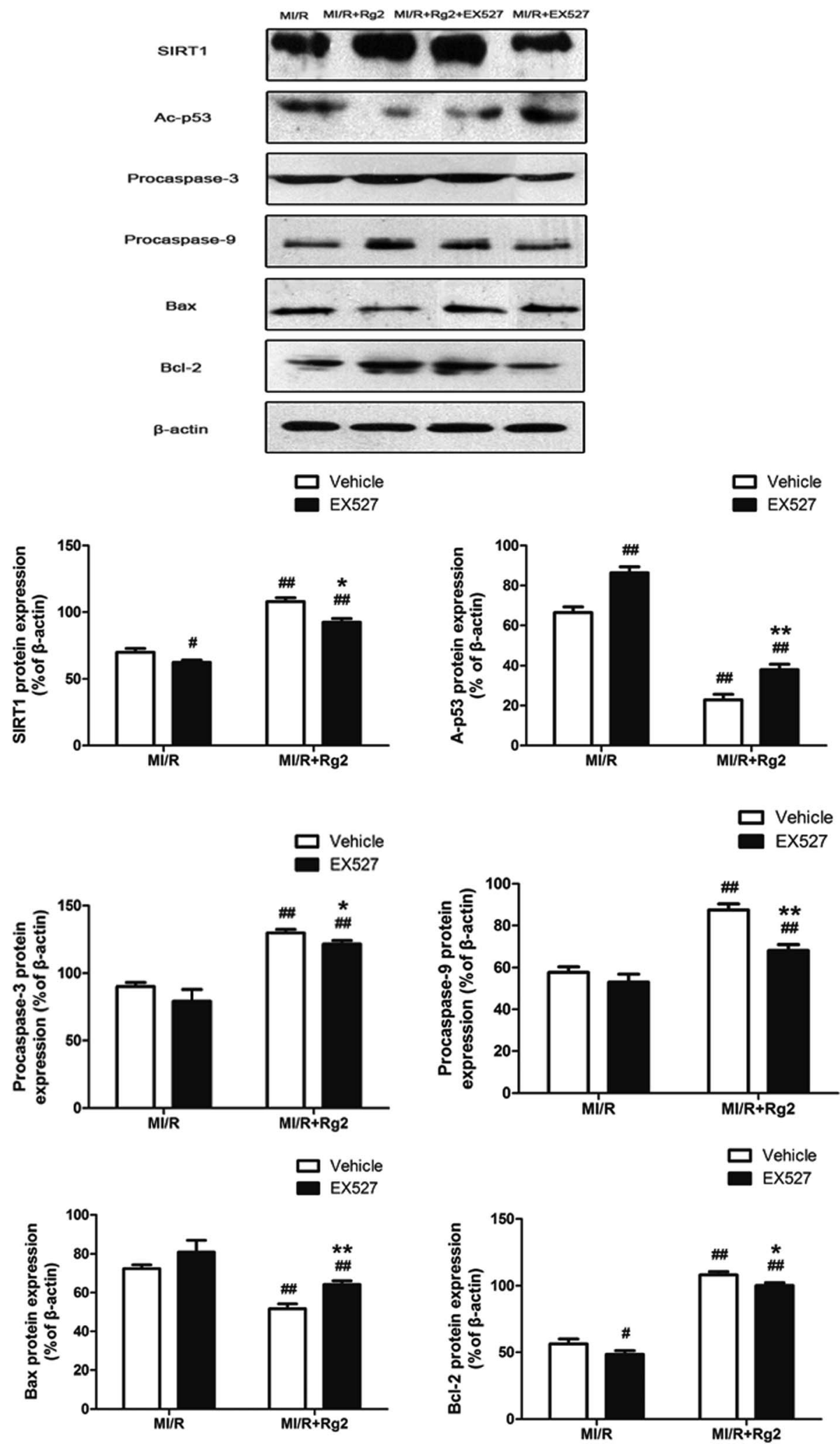

Fig. 11 Effects of 20(S)-ginsenoside Rg2 and EX527 on expression of SIRT1, Ac-p53, procaspase-3, procaspase-9, Bax and Bcl-2 in MI/Rinduced injury. SIRT1-related signaling and apoptosis-related proteins were measured after $6 \mathrm{~h}$ of reperfusion. Representative blots of expression of SIRT1, Ac-p53, procaspase-3, procaspase-9, Bax, and Bcl-2 and their evaluation are shown. Data are the mean \pm S.D. ${ }^{\#} P<0.05,{ }^{\# \#} P<0.01$ vs. MI/R group and $* P<0.05, * * P<0.01$ vs. MI/R + Rg2 group. 
$20(S)$-Ginsenoside $\mathrm{Rg} 2$ is a major active component in the roots and stem leaves of ginseng. 20(S)-Ginsenoside Rg2 has a wide range of pharmacologic effects. ${ }^{17,39-42}$ Our previous study demonstrated that $20(S)$-ginsenoside $\mathrm{Rg} 2$ can protect cardiomyocytes from $\mathrm{H}_{2} \mathrm{O}_{2}$-induced injury by inhibiting ROS production, increasing intracellular levels of antioxidants, and attenuating apoptosis. ${ }^{18}$ In the present study, we also found that pretreatment with $20(S)$-ginsenoside Rg2 conferred cardioprotective effects, as evidenced by improved post-MI/R cardiac function, attenuated oxidative stress and inflammation, as well as reduced myocardial apoptosis and necrosis.

We demonstrated that pretreatment with $20(S)$-ginsenoside Rg2 exerted antioxidative effects by decreasing cardiac superoxide generation, gp91 ${ }^{\text {phox }}$ expression, MDA content, as well as increasing the activities of SOD, CAT and GSH-PX. The mechanisms involved may be attributed to the scavenging of free radicals, increasing the concentration of antioxidants, and inhibiting cardiomyocyte apoptosis. In addition, pretreatment with $20(S)$-ginsenoside Rg2 exerted anti-inflammatory effects by decreasing the levels of IL- $1 \beta$, IL- 6 and TNF- $\alpha$. The mechanisms involved may be cell damage caused by the release of oxygen free radicals and cytotoxic substances. These released inflammatory mediators can damage vascular endothelial cells, increase vascular permeability, and further activate inflammatory cells, which increases the inflammatory response. ${ }^{43}$ Inhibition of myocardial oxidative stress and inflammation is very important for developing cardioprotective strategies against MI/ R injury. ${ }^{44}$

SIRT1 is a member of the class-III group of histone deacetylase, the activity of which is dependent on the intracellular ratio of $\mathrm{NAD}^{+} / \mathrm{NADH}$. In $\mathrm{MI} / \mathrm{R}$ injury, the deacetylase activity of SIRT1 has a key role in cardioprotection. ${ }^{22,45-47}$ It has been demonstrated that SIRT1 expression is reduced after MI/Rinduced injury, and that activation of SIRT1 signaling with curcumin, melatonin or berberine confers protection against MI/R-induced injury by suppressing apoptosis and oxidative stress. ${ }^{22-24,48}$ The present study is in accordance with our previous study in vitro (data not shown) demonstrating that $20(S)$-ginsenoside $\mathrm{Rg} 2$ can increase SIRT1 activity. It is also in accordance with a study showing that $20(S)$-ginsenoside Rg2 and some of its metabolites produced by liver microsomes in rats can activate SIRT1. ${ }^{31}$ However, whether the protective effect of $20(S)$-ginsenoside Rg2 is mediated by SIRT1 requires further investigation, so we employed a SIRT1 inhibitor. EX527 is a highly specific inhibitor of SIRT1, and it was used to suppress the change wrought by a potential activator of SIRT1: 20(S)ginsenoside $\mathrm{Rg} 2$. If EX527 could reverse the protective effects of 20(S)-ginsenoside Rg2, SIRT1 would be the curtailing protein that mediates the process. By employing EX527, the improvement in cardiac function provided by $20(S)$-ginsenoside Rg2 was abolished. EX527 also abrogated the protective effect of $20(S)$ ginsenoside $\mathrm{Rg} 2$ by increasing the apoptotic index, infarct size, as well as serum activities of CK-MB, AST and LDH, which aggravated apoptosis and necrosis.

Oxidative stress is a major contributor to primary and secondary MI/R injury. ${ }^{49}$ SIRT1 activity is dependent upon the intracellular ratio of $\mathrm{NAD}^{+} / \mathrm{NADH}$, which triggers it to respond

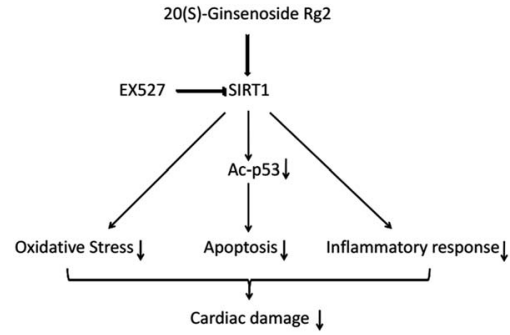

Fig. 12 Proposed cardioprotective signaling pathway of 20(S)-ginsenoside Rg2.

to the high level of oxidants. ${ }^{50}$ The transcription regulation and deacetylation observed upon SIRT1 administration can alleviate NADPH/NADH oxidase-derived ROS generation, ischemia and reperfusion. ${ }^{\mathbf{5 0 , 5 1}}$ We showed that EX527 increased superoxide generation, gp91 ${ }^{\text {phox }}$ expression and MDA content, but also decreased the activities of the antioxidant enzymes SOD, CAT and GSH-PX compared with the MI/R + Rg2 group. These results suggest that $20(S)$-ginsenoside $\mathrm{Rg} 2$ reduces post-MI/R oxidative stress, thereby improving cardiac function via a SIRT1-mediated network.

$\mathrm{MI} / \mathrm{R}$ injury is also probably related to myocardial inflammatory response due to the released pro-inflammatory mediators. The latter cause damage to vascular endothelial cells, increased vascular permeability, and activate immune cells which aggravate the inflammatory response. ${ }^{52}$ Studies have shown that SIRT1 activation reduces inflammatory damage. ${ }^{53,54}$ Here, we illustrated that pretreatment with $20(S)$-ginsenoside $\mathrm{Rg} 2$ reduced post-MI/R inflammation by decreasing the mRNA levels and activities of pro-inflammatory cytokines. Furthermore, EX527 abolished the effects of 20(S)-ginsenoside Rg2 on inflammation. Taken together with our previous study, SIRT1 is a curtailing mediator of $20(S)$-ginsenoside $\mathrm{Rg} 2$ that inhibits expression of pro-inflammatory cytokines.

SIRT1 regulates p53 acetylation, thereby inhibiting apoptosis of cardiomyocytes. ${ }^{19}$ Here, we found that $20(S)$-ginsenoside Rg2 induced a significant increase in SIRT1 expression and p53 deacetylation in the MI/R myocardium. Furthermore, 20 $(S)$ ginsenoside $\mathrm{Rg} 2$ increased expression of the anti-apoptotic factors Bcl-2, procaspase-3 and procaspase-9, and decreased expression of the pro-apoptotic factor Bax in MI/R-induced injury. However, the protective effects of $20(S)$-ginsenoside $\mathrm{Rg} 2$ were abolished by EX527. Therefore, 20(S)-ginsenoside Rg2 confers a protective effect against MI/R injury via SIRT1 by alleviating oxidative stress and reducing myocardial inflammation (Fig. 12).

\section{Conclusions}

20(S)-Ginsenoside $\mathrm{Rg} 2$ exerted a profound cardioprotective effect against $\mathrm{MI} / \mathrm{R}$ injury. This protection appeared to be due largely to activation of SIRT1 signaling and attenuation of oxidative stress and inflammation. Hence, 20(S)-ginsenoside Rg2 may be a promising agent for the treatment of MI/R injury in cardiac surgery and ischemic heart diseases. 


\section{Conflicts of interest}

The authors declare no conflict of interests.

\section{Funding}

The authors gratefully acknowledge financial support from the National Natural Science Foundation of China (81473378) and Natural Science Foundation of Jilin Province (20170101002JC).

\section{Acknowledgements}

The authors thank Dr Yifa Zhou for providing 20(S)-ginsenoside Rg2 and Dr Tian Wang for his critical reading and language editing of this manuscript.

\section{References}

1 A. Sekikawa, B. Y. Horiuchi, D. Edmundowicz, H. Ueshima, J. D. Curb, K. Sutton-Tyrrell, T. Okamura, T. Kadowaki, A. Kashiwagi, K. Mitsunami, K. Murata, Y. Nakamura, B. L. Rodriguez and L. H. Kuller, Heart, 2003, 89, 255-257.

2 S. Sanada, I. Komuro and M. Kitakaze, Am. J. Physiol.: Heart Circ. Physiol., 2011, 301, H1723-H1741.

3 K. Matsumura, R. W. Jeremy, J. Schaper and L. C. Becker, Circulation, 1998, 97, 795-804.

4 J. L. Zweier and M. A. Talukder, Cardiovasc. Res., 2006, 70, 181-190.

5 Z. Q. Zhao, Curr. Opin. Pharmacol., 2004, 4, 159-165.

6 R. A. Gottlieb, J. Cardiovasc. Pharmacol. Ther., 2011, 16, 233238.

7 G. Petrosillo, F. M. Ruggiero, N. Di Venosa and G. Paradies, FASEB J., 2003, 17, 714-716.

8 D. B. Zorov, C. R. Filburn, L. O. Klotz, J. L. Zweier and S. J. Sollott, J. Exp. Med., 2000, 192, 1001-1014.

9 C. Maack and B. O'Rourke, Basic Res. Cardiol., 2007, 102, 369-392.

$10 \mathrm{H}$. Tsutsui, T. Ide and S. Kinugawa, Antioxid. Redox Signaling, 2006, 8, 1737-1744.

11 K. W. Leung and A. S. Wong, Chin. Med., 2010, 5, 20.

12 S. Chae, K. A. Kang, W. Y. Chang, M. J. Kim, S. J. Lee, Y. S. Lee, H. S. Kim, D. H. Kim and J. W. Hyun, J. Agric. Food Chem., 2009, 57, 5777-5782.

13 W. C. Cho, W. S. Chung, S. K. Lee, A. W. Leung, C. H. Cheng and K. K. Yue, Eur. J. Pharmacol., 2006, 550, 173-179.

14 W. Wang, E. R. Rayburn, M. Hao, Y. Zhao, D. L. Hill, R. Zhang and H. Wang, Prostate, 2008, 68, 809-819.

15 C. Mancuso and R. Santangelo, Food Chem. Toxicol., 2017, 107, 362-372.

16 X. W. Yang, L. Y. Ma, Q. L. Zhou, W. Xu and Y. B. Zhang, Bioorg. Med. Chem. Lett., 2017, 28, 240-243.

17 Y. Jiang, W. Liu, X. M. Wang, G. G. Zhong, W. J. Zhang, L. Chen, S. Zhan, H. Qi, C. Y. Zhao, X. Y. Ma, S. J. Yang and H. Li, Zhongguo Yaoli Xuebao, 1996, 17, 138-141.

18 W. Fu, D. Sui, X. Yu, D. Gou, Y. Zhou and H. Xu, Int. J. Clin. Exp. Med., 2015, 8, 19938-19947.
19 R. R. Alcendor, L. A. Kirshenbaum, S. Imai, S. F. Vatner and J. Sadoshima, Circ. Res., 2004, 95, 971-980.

20 C. P. Hsu, P. Y. Zhai, T. Yamamoto, Y. Maejima, S. Matsushima, N. Hariharan, D. Shao, H. Takagi, S. Oka and J. Sadoshima, Circulation, 2010, 122, 2170-U2193.

21 C. Tong, A. Morrison, S. Mattison, S. Qian, M. Bryniarski, B. Rankin, J. Wang, D. P. Thomas and J. Li, FASEB J., 2013, 27, 4332-4342.

22 Y. Yang, W. Duan, Y. Lin, W. Yi, Z. Liang, J. Yan, N. Wang, C. Deng, S. Zhang, Y. Li, W. Chen, S. Yu, D. Yi and Z. Jin, Free Radical Biol. Med., 2013, 65, 667-679.

23 L. Yu, Q. Li, B. Yu, Y. Yang, Z. Jin, W. Duan, G. Zhao, M. Zhai, L. Liu, D. Yi, M. Chen and S. Yu, Oxid. Med. Cell. Longevity, 2016, 2016, 1689602.

24 L. Yu, Y. Sun, L. Cheng, Z. Jin, Y. Yang, M. Zhai, H. Pei, X. Wang, H. Zhang, Q. Meng, Y. Zhang, S. Yu and W. Duan, J. Pineal Res., 2014, 57, 228-238.

25 D. H. Kim, C. H. Park, D. Park, Y. J. Choi, M. H. Park, K. W. Chung, S. R. Kim, J. S. Lee and H. Y. Chung, Arch. Pharmacal Res., 2014, 37, 813-820.

26 Z. Song, Y. Liu, B. Hao, S. Yu, H. Zhang, D. Liu, B. Zhou, L. Wu, M. Wang, Z. Xiong, C. Wu, J. Zhu and X. Qian, PLoS One, 2014, 9, e112699.

27 Y. Y. Lee, J. S. Park, E. J. Lee, S. Y. Lee, D. H. Kim, J. L. Kang and H. S. Kim, J. Agric. Food Chem., 2015, 63, 3472-3480.

28 Y. Wang, Y. Chen, H. Wang, Y. Cheng and X. Zhao, Anal. Chem., 2015, 87, 5046-5049.

29 J. L. Yang, T. K. Q. Ha, B. Dhodary, K. H. Kim, J. Park, C. H. Lee, Y. C. Kim and W. K. Oh, J. Nat. Prod., 2014, 77, 1615-1623.

30 J. Y. Han, S. Lee, J. H. Yang, S. Kim, J. Sim, M. G. Kim, T. C. Jeong, S. K. Ku, I. J. Cho and S. H. Ki, J. Ginseng Res., 2015, 39, 105-115.

31 L. Y. Ma, Q. L. Zhou, X. B. Yang, H. P. Wang and X. W. Yang, Molecules, 2016, 21, 757.

32 J. M. Solomon, R. Pasupuleti, L. Xu, T. McDonagh, R. Curtis, P. S. DiStefano and L. J. Huber, Mol. Cell. Biol., 2006, 26, 2838.

33 Y. H. Jia, Z. Zheng, Y. C. Wang, Q. Zhou, W. X. Cai, W. B. Jia, L. L. Yang, M. L. Dong, X. X. Zhu, L. L. Su and D. H. Hu, PLoS One, 2015, 10, e0120849.

34 X. Ji, B. K. Tan, Y. C. Zhu, W. Linz and Y. Z. Zhu, Life Sci., 2003, 73, 1413-1426.

35 S. H. Lim and J. Lee, Prev. Nutr. Food Sci., 2012, 17, 177-183. 36 H. Su, L. Ji, W. Xing, W. Zhang, H. Zhou, X. Qian, X. Wang, F. Gao, X. Sun and H. Zhang, J. Cell. Mol. Med., 2013, 17, 181191.

37 J. Lin, H. Wang, J. Li, Q. Wang, S. Zhang, N. Feng, R. Fan and J. Pei, Cytokine, 2013, 61, 842-848.

38 X. Wu, B. Zhang, R. Fan, L. Zhao, Y. Wang, S. Zhang, A. D. Kaye, L. Huang and J. Pei, Cytokine, 2011, 56, 503-507.

39 S. Choi, S. Y. Jung, J. H. Lee, F. Sala, M. Criado, J. Mulet, L. M. Valor, S. Sala, A. G. Engel and S. Y. Nah, Eur. J. Pharmacol., 2002, 442, 37-45.

40 K. Kudo, E. Tachikawa, T. Kashimoto and E. Takahashi, Eur. J. Pharmacol., 1998, 341, 139-144. 
41 F. Sala, J. Mulet, S. Choi, S. Y. Jung, S. Y. Nah, H. Rhim, L. M. Valor, M. Criado and S. Sala, J. Pharmacol. Exp. Ther., 2002, 301, 1052-1059.

42 E. Tachikawa, K. Kudo, T. Kashimoto and E. Takahashi, J. Pharmacol. Exp. Ther., 1995, 273, 629-636.

43 X.-L. M. A. M. Lefer, A. Weyrich and D. J. Lefer, Agents Actions Suppl., 1993, 41, 127-135.

44 Y. Tian, H. Li, P. Liu, J. M. Xu, M. G. Irwin, Z. Xia and G. Tian, Mediators Inflammation, 2015, 2015, 819232.

45 C. J. Chen, W. Yu, Y. C. Fu, X. Wang, J. L. Li and W. Wang, Biochem. Biophys. Res. Commun., 2009, 378, 389-393.

46 S. Chung, H. Yao, S. Caito, J. W. Hwang, G. Arunachalam and I. Rahman, Arch. Biochem. Biophys., 2010, 501, 79-90.

47 C. P. Hsu, P. Zhai, T. Yamamoto, Y. Maejima, S. Matsushima, N. Hariharan, D. Shao, H. Takagi, S. Oka and J. Sadoshima, Circulation, 2010, 122, 2170-2182.
48 L. Yu, F. Li, G. Zhao, Y. Yang, Z. Jin, M. Zhai, W. Yu, L. Zhao, W. Chen, W. Duan and S. Yu, Apoptosis, 2015, 20, 796-810.

49 H. Li, Y. Bian, N. Zhang, J. Guo, C. Wang, W. B. Lau and C. Xiao, Cardiovasc. Diabetol., 2013, 12, 91.

50 S. Y. Shin, T. H. Kim, H. Wu, Y. H. Choi and S. G. Kim, Eur. J. Pharmacol., 2014, 727, 115-124.

51 T. Zhang, J. G. Berrocal, K. M. Frizzell, M. J. Gamble, M. E. DuMond, R. Krishnakumar, T. L. Yang, A. A. Sauve and W. L. Kraus, J. Biol. Chem., 2009, 284, 20408-20417.

52 A. M. Lefer, X. L. Ma, A. Weyrich and D. J. Lefer, Agents Actions Suppl., 1993, 41, 127-135.

53 S. Winnik, J. Auwerx, D. A. Sinclair and C. M. Matter, Eur. Heart J., 2015, 36, 3404-3412.

54 Y. Zhang, X. X. Liu, Z. G. She, D. S. Jiang, N. A. Wan, H. Xia, X. H. Zhu, X. Wei, X. D. Zhang and H. L. Li, Basic Res. Cardiol., 2014, 109, 434. 